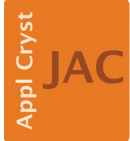

JOURNAL OF

APPLIED

CRYSTALLOGRAPHY

ISSN 1600-5767

Received 11 April 2016

Accepted 23 May 2016

Edited by V. Holý, Charles University, Prague, Czech Republic

Keywords: X-ray dynamical diffraction theory; lateral structures; lateral crystals; reciprocal space maps; Bragg-Laue geometry; coherent diffraction imaging; deformations.

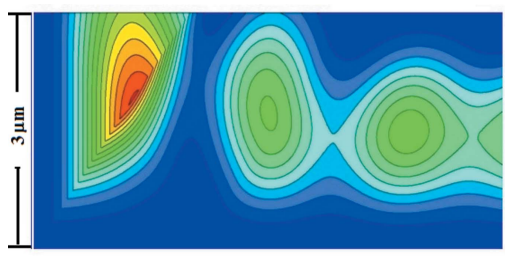

(C) 2016 International Union of Crystallography

\section{Bragg-Laue X-ray dynamical diffraction on perfect and deformed lateral crystalline structures}

\author{
Vasily I. Punegov, ${ }^{\mathrm{a}, \mathrm{b} *}$ Sergey I. Kolosov ${ }^{\mathrm{a}}$ and Konstantin M. Pavlov ${ }^{\mathrm{c}, \mathrm{d}}$ \\ ${ }^{\mathbf{a}}$ Komi Research Center, Ural Division, Russian Academy of Sciences, Syktyvkar, 167982, Russian Federation, ${ }^{\mathbf{b}}$ Syktyvkar \\ State University, Syktyvkar, 167001, Russian Federation, 'SChool of Science and Technology, University of New England, \\ NSW 2351, Australia, and ${ }^{\mathbf{d} S}$ chool of Physics and Astronomy, Monash University, VIC 3800, Australia. *Correspondence \\ e-mail: vpunegov@dm.komisc.ru
}

The new dynamical diffraction approach to X-ray diffraction on lateral crystalline structures has been developed to investigate the angular and spatial distribution of wavefields in the case of the Bragg-Laue geometry in non-perfect lateral structures. This approach allows one to calculate reciprocal space maps for deformed lateral crystals having rectangular cross sections for both the transmitted and reflected wavefields. Numerical modelling is performed for crystals with different lateral sizes, thicknesses and deformations. The approach can be used in coherent diffraction imaging to simulate Fraunhofer diffraction patterns produced by relatively large deformed crystals.

\section{Introduction}

Lateral (having a finite length in the lateral direction) crystalline structures (e.g. nanowires) are widely used in opto- and nanoelectronics. For instance, core-shell nanowires, having a high surface-to-volume ratio, are promising candidates for light emitting diodes (Yan et al., 2009), solar cells (Krogstrup et al., 2013), transistors (Colinge et al., 2010) and other devices (Li et al., 2006; Dasgupta et al., 2014). In recent years there has been a renewed interest in using X-ray diffraction as a characterization technique on such lateral structures (see e.g. Kaganer \& Belov, 2012; Minkevich et al., 2011; Lee et al., 2006; Stankevič et al., 2015, and references therein). Lateral structures are also often the objects of interest (see e.g. Cha et al., 2010; Köhl et al., 2013; Yang et al., 2013) in applications of coherent diffraction imaging (CDI) [see a recent review of Bragg CDI by Vartanyants \& Yefanov (2015) and references therein].

Some dynamical diffraction approaches (Olekhnovich \& Olekhnovich, 1978; Thorkildsen \& Larsen, 1999a; Kolosov \& Punegov, 2005; Yan \& Li, 2014) based on Takagi's equations (Takagi, 1962, 1969) have been employed to calculate rocking curves from ideal (i.e. non-deformed) crystals with a rectangular cross section. A simpler kinematical diffraction theory was used to simulate X-ray diffraction on deformed crystals having a trapezium cross-sectional shape (Punegov et al., 2006; Punegov \& Kolosov, 2007) or an arbitrary cross-sectional shape (Punegov et al., 2007). However, this does not take into account dynamical diffraction effects, which become evident even for relatively small crystals (Punegov et al., 2014).

Usually, Takagi's equations are used to simulate dynamical X-ray diffraction on lateral crystalline structures (Becker, 1977; Becker \& Dunstetter, 1984; Olekhnovich \& Olekhnovich, 1980; Saldin, 1982; Chukhovskii et al., 1998; Thorkildsen \& Larsen, 1999b). However, these approaches do not allow 
one to simulate reciprocal space maps (RSMs). Recently, we applied (Punegov et al., 2014) Darwin's recurrence relations to calculate the crystal rocking curves and RSMs in Bragg geometry for X-ray diffraction on non-deformed lateral crystals. However, a simultaneous Bragg and Laue or a mixed Laue-Bragg geometry is a more realistic variant for lateral crystals.

Lehmann \& Borrmann (1967, and references therein) observed, explained and later numerically modelled interference fringes appearing in Laue-Bragg geometry. The experiment and calculations were done by Lehmann \& Borrmann for a point source placed at the crystal surface. The partial reflection at one of the crystal sides (which is parallel to the crystal reflection plane) produces an additional 'virtual' source, shifted with respect to the real one. Such a 'virtual' source, caused by reflection, is often used in optics, for instance as Lloyd's mirror (Born \& Wolf, 1999; Jesson et al., 2007), to produce interference fringes.

Saka and co-workers generalized the approach presented by Lehmann \& Borrmann (1967) and discussed the theory of X-ray diffraction for finite polyhedral crystals in the LaueBragg (Saka et al., 1972a), Laue-(Bragg) ${ }^{\mathrm{m}}$ (Saka et al., 1972b, 1973) and Bragg-(Bragg) ${ }^{\mathrm{m}}$ (Saka et al., 1973) cases. In particular, Kato's dynamical diffraction theory (Kato, 1961a,b) for spherical waves (represented as a superposition of plane waves) was employed. Saka et al. (1972a) also defined four special cases - Laue-Laue, Bragg-Bragg, Laue-Bragg and Bragg-Laue - for different configurations for incident and exit waves with respect to the entrance and exit surfaces of a crystal. For instance, they said (Saka et al., 1972a, p. 103) that 'when the Bragg reflected waves emerge from the entrance surface, we shall call this the 'Bragg case on the entrance surface', whereas when they all propagate through the crystal, the case is called the "Laue case on the entrance surface". It should be noted that Saka et al. (1972a) did not consider the case of a lateral crystal which we discuss in this paper. For a lateral crystal it is possible (see $\$ 3$ below) to have simultaneously both the above-mentioned cases (namely, Laue and Bragg cases) for the entrance surface. Similarly, both Laue and Bragg cases can be observed for the exit surface in lateral crystals (see $\$ 3$ below). Saka and co-workers also considered a finite polyhedral crystal (Saka et al., 1972b, 1973), but only for the case of a spherical incident wave, having a limited wavefront at the entrance surface. This differs significantly from the configuration discussed in this paper, where we deal with deformed lateral crystals 'bathing' in the incident plane wave and calculate the distribution of diffracted intensity in reciprocal space.

While in their original study Lehmann and Borrmann considered the strong absorption case ( $\mu t>18$ ), where $\mu$ is the normal X-ray linear absorption coefficient and $t$ the crystal thickness, Mai \& Zhao (1989) discussed in detail BorrmannLehmann interference in the moderate absorption case $(\mu t=$ 1.6). In particular, they noted that the observed fringe spacing in the case of low or moderate absorption is not described by formulae originally obtained for the case of strong absorption. Mai \& Zhao (1989) derived a general formula of the observed fringe spacing which is valid for high, moderate and low absorption. Their computer simulation was in good agreement with the experimental topographs.

Lang et al. $(1986,1990)$ used synchrotron radiation to study the Borrmann-Lehmann interference effect. They observed a high sensitivity of the interference fringes to lattice distortion. Such a high sensitivity can be qualitatively explained by analogy with X-ray interferometry. Their modelling (Lang et al., 1990) was based on Kato's ray-optical diffraction theory for a mildly distorted crystal (Kato, 1963, 1964a,b) with the assumption of a constant strain gradient.

Uragami (1983) numerically simulated the intensity distribution of the wavefield, caused by a very narrow incident wave, in an ideal rectangular-shaped crystal, using the Takagi equations (Takagi, 1962,1969) in the Laue geometry case. The result of the numerical simulation was compared with an analytical solution (Uragami, 1971) using a Green's function approach (Uragami, 1969, 1970; Afanas'ev \& Kohn, 1971). It was demonstrated that the transmitted wave, upon hitting a side face of the rectangular-shaped crystal, is reflected back inside the crystal and then interferes with the Bragg diffracted wave already propagating in the crystal. However, the distribution of intensity in reciprocal space, discussed in this paper, was not investigated. Also, in addition to a different calculation algorithm, we consider an incident plane wave instead of a delta function like the narrow incident wave used by Uragami (1983).

Yan \& Noyan (2005) investigated an effect of dynamical diffraction for a spatially limited and slightly divergent (i.e. non-parallel) beam by a plane-parallel Si crystal in the BraggLaue, Bragg-Bragg-Laue and multiple Bragg-Bragg cases. In particular, they observed fictitious double spots at the spatial detector caused by back-surface diffraction. Additionally, they provided a set of equations that can be used to calculate the separation of such peak pairs. Such double peaks in Laue diffraction patterns may cause difficulties in the strain analysis, as shown in their subsequent paper (Yan \& Noyan, 2006). To avoid such a problem, an additional (analyser) crystal in the diffracted beam can be used (Yan \& Noyan, 2006). This analyser crystal allows one to distinguish spatial and angular shifts of the diffracted beam.

It should be noted that the method first proposed by us in 2014 (Punegov et al., 2014) and further developed in this paper might seem similar to the numerical calculations of images in X-ray topography (see e.g. Authier et al., 1968; Taupin, 1967; Bowen \& Tanner, 1998; Authier, 2001; Epelboin, 1985). However, our method differs from the above in that it deals with lateral crystals 'bathing' in the incident plane wave and allows one to calculate the distribution of diffracted intensity in reciprocal space.

Currently, the kinematical diffraction approach is used to simulate a far-field diffraction pattern in CDI (see Vartanyants \& Yefanov, 2015, and references therein). However, as correctly mentioned by Vartanyants \& Yefanov (2015, p. 346), 'if crystalline particles reach micron size, multiple scattering, or dynamical effects ... could become important ...'. Modern synchrotron sources are already able to achieve a coherence 
volume of the order of several micrometres (Pellegrini \& Stöhr, 2003). If the illuminated object, having a size of several micrometres, matches the coherence volume, the dynamical diffraction effects (Authier, 2001) start to be observable. For instance, the Pendellösung oscillations in the Laue diffraction geometry (see $\$ 3$ below) cannot be simulated or reconstructed using kinematical diffraction theory. However, the current variants of dynamical diffraction on lateral crystalline structures (exclusive of Punegov et al., 2014) do not allow the simulation of RSMs, which are required in CDI for the reconstruction of the electron density and deformation field in the sample. Also, the variant of dynamical diffraction theory developed by Punegov et al. (2014) did not take into account the deformation field in the lateral crystal, which is often the most important part of the information to be reconstructed by CDI. Therefore, it is timely to explore the possibilities offered by dynamical diffraction on deformed lateral crystals in application to CDI. This will make it possible to employ the new capabilities (e.g. large coherence volume) offered by modern synchrotron sources.

The purpose of the current paper is to present a new approach to dynamical diffraction theory based on Takagi's equations in the case of Bragg-Laue geometry for deformed lateral crystals. This approach allows one to effectively calculate RSMs for deformed lateral crystals having rectangular cross sections for both the transmitted and reflected

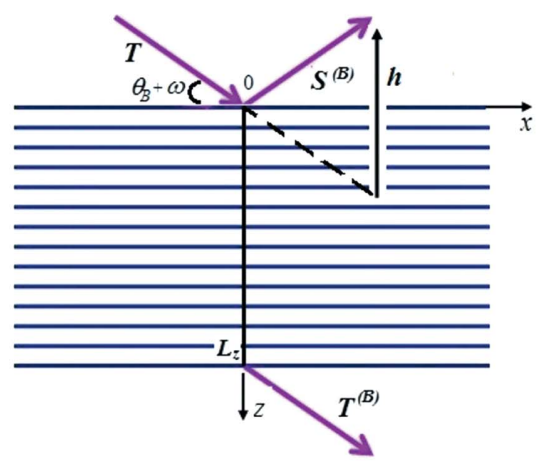

(a)

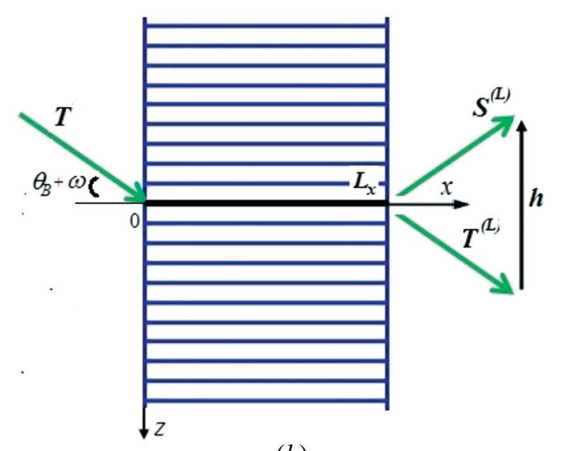

(b)

Figure 1

A schematic of the symmetrical Bragg (a) and Laue (b) X-ray diffraction by a crystal having the same family of reflecting atomic planes $(h k l)$, corresponding to the reciprocal lattice vector $\mathbf{h}$. wavefields along with the intensity distribution within the crystal.

\section{Dynamical diffraction on lateral crystalline structures with a rectangular cross section}

The current variants of X-ray dynamical diffraction theory (Authier, 2001) usually consider crystals with the shapes of plane-parallel slabs (see Fig. 1 and Appendix $A$ for details) having finite thickness only in one direction. In this paper we consider lateral crystalline structures with a rectangular cross section (see Fig. 2) having a finite width of $L_{x}$ and thickness of $L_{z}$.

We consider simultaneously both geometries, namely the Bragg and Laue geometries (see Fig. 2). Therefore, this case can be classified as mixed Bragg-Laue diffraction, and we calculate both the transmitted and diffracted wavefields taking into account their appropriate boundary conditions at all four external surfaces of a lateral crystal. Fig. 2 demonstrates the local areas of either Bragg or Laue diffraction on such a lateral crystal.

Let us introduce a dynamical extension of the kinematical approach, previously reported by Kirste et al. (2005). We consider two-beam diffraction in the coplanar geometry, where the $x 0 y$ plane is the top surface of a lateral crystal and the $x 0 z$ plane is the plane of diffraction (see Fig. 2). The incident wave is assumed to be a plane monochromatic wave. A solution of Maxwell's equations (see e.g. Afanas'ev \& Kohn, 1971) within the crystal is sought in the following form:

$$
\mathbf{E}(\mathbf{r})=\mathbf{E}_{0}(\mathbf{r}) \exp \left(i \mathbf{k}_{0}^{\mathrm{B}} \cdot \mathbf{r}\right)+\mathbf{E}_{h}(\mathbf{r}) \exp \left[i\left(\mathbf{k}_{0}^{\mathrm{B}}+\mathbf{h}\right) \cdot \mathbf{r}\right],
$$

where, following Takagi (see e.g. Takagi, 1969), we assume that the amplitudes of the transmitted and diffracted waves $\mathbf{E}_{0, h}(\mathbf{r})$ are slowly varying functions. The vector $\mathbf{k}_{0}^{\mathrm{B}}$ can be chosen arbitrarily. In our case this vector is defined as always satisfying the Bragg conditions and its magnitude is $2 \pi / \lambda$, where $\lambda$ is the X-ray wavelength in vacuum. The vector $\mathbf{h}$ is the appropriate vector of the reciprocal lattice (see Fig. 2).

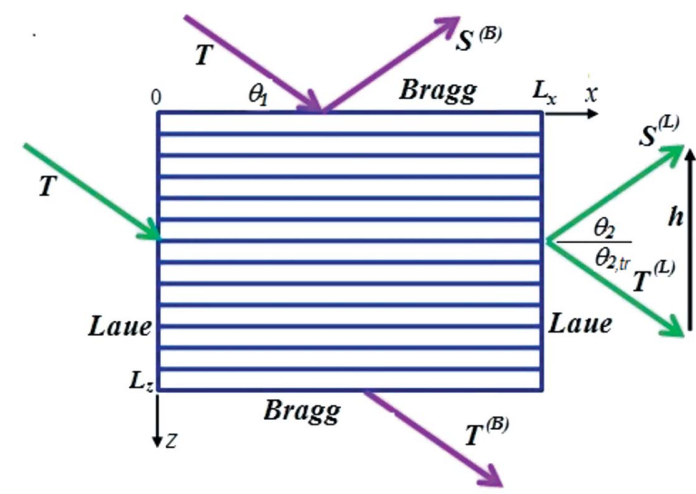

Figure 2

A schematic of the Bragg-Laue diffraction on a lateral crystal of a rectangular cross section. The reciprocal vector $\mathbf{h}$ corresponds to the family $(h k l)$ of reflection planes. 
Inside the crystal the X-ray wavefield is described by a system of equations of the dynamical diffraction based on Takagi's equations (Takagi, 1969; Afanas'ev \& Kohn, 1971):

$$
\begin{aligned}
& \frac{\partial E_{0}(\mathbf{r})}{\partial s_{0}}=i \frac{\pi \chi_{0}(\mathbf{r})}{\lambda} E_{0}(\mathbf{r})+i \frac{\pi \chi_{\bar{h}}(\mathbf{r}) C}{\lambda} E_{h}(\mathbf{r}) \exp [i \mathbf{h} \cdot \mathbf{u}(\mathbf{r})], \\
& \frac{\partial E_{h}(\mathbf{r})}{\partial s_{h}}=i \frac{\pi \chi_{0}(\mathbf{r})}{\lambda} E_{h}(\mathbf{r})+i \frac{\pi \chi_{h}(\mathbf{r}) C}{\lambda} E_{0}(\mathbf{r}) \exp [-i \mathbf{h} \cdot \mathbf{u}(\mathbf{r})] .
\end{aligned}
$$

Here $\chi_{0, h, \bar{h}}$ are the Fourier components of the susceptibility, $C$ is the polarization factor and $\mathbf{u}$ is the atomic displacement vector. The functions $\chi_{0, h, \bar{h}}$ depend on $\mathbf{r}$ owing to the variation in the chemical composition. It should be noted that in (2) we use the expressions for $\chi_{0, h, \bar{h}}$ obtained for an infinitely large crystal, which is, obviously, an approximation for a lateral crystal. The $s_{0}$ coordinate axis is directed along the vector $\mathbf{k}_{0}^{\mathrm{B}}$ and the $s_{h}$ coordinate axis is directed along the vector $\mathbf{k}_{0}^{\mathrm{B}}+\mathbf{h}$. Although (2) describes a general case of asymmetrical diffraction and arbitrary polarization, in this paper, for the sake of simplicity, we consider only the symmetrical coplanar diffraction of $\sigma$-polarized waves by a lateral crystal of rectangular shape. Such a shape belongs to the so-called convex polygons that contain all the line segments connecting any pair of its points.

The wavefield $\mathbf{E}^{v}(\mathbf{r})$ just outside the crystal volume can be represented as a combination of the incident, diffracted and transmitted waves, respectively:

$$
\begin{aligned}
\mathbf{E}^{v}(\mathbf{r})= & \mathbf{E}_{0}^{v}(\mathbf{r}) \exp \left(i \mathbf{k}_{0}^{v} \cdot \mathbf{r}\right)+\mathbf{E}_{h}^{v}(\mathbf{r}) \exp \left(i \mathbf{k}_{h}^{v} \cdot \mathbf{r}\right) \\
& +\mathbf{E}_{\mathrm{tr}}^{v}(\mathbf{r}) \exp \left(i \mathbf{k}_{\mathrm{tr}}^{v} \cdot \mathbf{r}\right) .
\end{aligned}
$$

Here $\mathbf{k}_{0}^{v}$ is the average wavevector of the incident wave, formed by the X-ray source and optical system before the lateral crystal, and $\mathbf{k}_{h, \text { tr }}^{v}$ are the average wavevectors of the diffracted and transmitted waves selected either spatially (e.g. by a two-dimensional detector placed at a large distance after the lateral crystal) or angularly by an analyser crystal. We consider a lateral crystal which is 'bathing' in the incident plane wave. Thus, the incident wave consists of two parts. The first interacts with the lateral crystal and the second does not. The latter can be excluded from the registration process, for instance, by slits, which will cut off the part of the incident wave that does not interact with the crystal. Then the wavefields $\mathbf{E}_{0}^{v}(\mathbf{r})$ and $\mathbf{E}_{\mathrm{tr}}^{v}(\mathbf{r})$ are spatially separated. The first is located at the vertical left face and top surface of the crystal (i.e. in the space before the interaction with the lateral crystal occurs), and the second exists in the crystal 'shadow': at the right vertical face and the bottom surface of the crystal (i.e. after the propagated wavefield has interacted with the crystal) (see Fig. 2).

In this paper we assume that the incident wave can be approximated by a monochromatic plane wave, which is a reasonable approximation for modern synchrotron sources if monochromator crystals are used and the distance between the source and the sample is relatively large. Then $\mathbf{E}_{0}^{v}(\mathbf{r})$ is a constant. However, this is not true for the transmitted and diffracted amplitudes. The amplitudes $\mathbf{E}_{h}^{v}(\mathbf{r})$ and $\mathbf{E}_{\mathrm{tr}}^{v}(\mathbf{r})$ are a position-dependent function and can be calculated using recurrence relations, which will be introduced below. Along with the traditionally used scattering vector $\mathbf{Q}=\mathbf{k}_{h}^{v}-\mathbf{k}_{0}^{v}$, usually used to describe the distribution of the diffracted intensity in reciprocal space, we can also define a 'transmission' scattering vector $\mathbf{Q}_{\mathrm{tr}}=\mathbf{k}_{\mathrm{tr}}^{v}-\mathbf{k}_{0}^{v}$ to describe the deviation of the transmitted wavefield from the original direction, $\mathbf{k}_{0}^{v}$, of the incident wave.

Let us assume that the experimental setup uses an analyser crystal with a very narrow (like a delta function) rocking curve to select a particular direction in reciprocal (Fourier) space, defined by either $\mathbf{k}_{h}^{v}$ (diffracted wave) or $\mathbf{k}_{\mathrm{tr}}^{v}$ (transmitted wave). The wave reflected by such an analyser crystal is then integrated by a point detector. It was shown by Pavlov et al. (2001, see equation 2 therein) that the intensity registered by such a point detector is proportional to the integral over the beam cross section for the propagating wavefield just after the object. In our experimental setup this integral can be approximated by the absolute square of a sum over the crystal surface for either $\mathbf{E}_{h}^{v}(\mathbf{r})$ or $\mathbf{E}_{\mathrm{tr}}^{v}(\mathbf{r})$ for the diffracted and transmitted waves, respectively. The same approximation can be used for a typical CDI experimental setup, which usually uses a two-dimensional position-sensitive detector placed in the far-field zone.

The information about the deviation, $\mathbf{q}$ ( or $\mathbf{q}_{\mathrm{tr}}$ ), of the scattering vector, $\mathbf{Q}$ ( or $\mathbf{Q}_{\mathrm{tr}}$ ), from the vector $\mathbf{h}$ (or zero for the transmitted beam) consists of the boundary conditions at the external surfaces of the lateral crystal [see $(4 a),(4 b),(4 c),(4 d)$ below].

We assume that the crystal has a rectangular shape (see Fig. 2); then we can write four boundary conditions at the following external surfaces:

(a) At the top surface and left vertical face of the crystal (for the incoming incident wave)

$$
E_{0}^{v}\left(\mathbf{r}_{e}\right) \exp \left(i \mathbf{k}_{0}^{v} \cdot \mathbf{r}_{e}\right)=E_{0}\left(\mathbf{r}_{e}\right) \exp \left(i \mathbf{k}_{0}^{\mathrm{B}} \cdot \mathbf{r}_{e}\right)
$$

(b) At the top surface and right vertical face of the crystal (for the leaving diffracted wave)

$$
E_{h}^{v}\left(\mathbf{r}_{e}\right) \exp \left(i \mathbf{k}_{h}^{v} \cdot \mathbf{r}_{e}\right)=E_{h}\left(\mathbf{r}_{e}\right) \exp \left[i\left(\mathbf{k}_{0}^{\mathrm{B}}+\mathbf{h}\right) \cdot \mathbf{r}_{e}\right] .
$$

(c) At the bottom surface and right vertical face of the crystal (for the leaving transmitted wave)

$$
E_{\mathrm{tr}}^{v}\left(\mathbf{r}_{e}\right) \exp \left(i \mathbf{k}_{\mathrm{tr}}^{v} \cdot \mathbf{r}_{e}\right)=E_{0}\left(\mathbf{r}_{e}\right) \exp \left(i \mathbf{k}_{0}^{\mathrm{B}} \cdot \mathbf{r}_{e}\right)
$$

(d) At the left vertical face and bottom surface of the crystal (to indicate the absence of the incoming diffracted wave from this particular direction)

$$
0=E_{h}\left(\mathbf{r}_{e}\right) \exp \left[i\left(\mathbf{k}_{0}^{\mathrm{B}}+\mathbf{h}\right) \cdot \mathbf{r}_{e}\right]
$$

Here $\mathbf{r}_{e}$ is the vector defining a position at the crystal surface. Because of the coplanar diffraction case, the $y$ component for all wavevectors in $(4 a),(4 b),(4 c),(4 d)$ is equal to 0 . The projections of the wavevectors $\mathbf{k}_{0, h, \text { tr }}^{v}$ are as follows: 


$$
\begin{aligned}
& k_{0, x}^{v}=k \cos \left(\theta_{1}\right)=k \cos \left(\theta_{\mathrm{B}}+\Delta \theta_{1}\right), \\
& k_{0, z}^{v}=k \sin \left(\theta_{1}\right)=k \sin \left(\theta_{\mathrm{B}}+\Delta \theta_{1}\right) ; \\
& k_{h, x}^{v}=k \cos \left(\theta_{2}\right)=k \cos \left(\theta_{\mathrm{B}}+\Delta \theta_{2}\right), \\
& k_{h, z}^{v}=-k \sin \left(\theta_{2}\right)=-k \sin \left(\theta_{\mathrm{B}}+\Delta \theta_{2}\right) ; \\
& k_{\mathrm{tr}, x}^{v}=k \cos \left(\theta_{2, \mathrm{tr}}\right)=k \cos \left(\theta_{\mathrm{B}}+\Delta \theta_{2, \mathrm{tr}}\right), \\
& k_{\mathrm{tr}, z}^{v}=k \sin \left(\theta_{2, \mathrm{tr}}\right)=k \sin \left(\theta_{\mathrm{B}}+\Delta \theta_{2, \mathrm{rr}}\right)
\end{aligned}
$$

Here $\Delta \theta_{1}, \Delta \theta_{2}$ and $\Delta \theta_{2, \text { tr }}$ are small deviations from the exact Bragg angular position determined by $\theta_{\mathrm{B}}$. Their positive/ negative directions are defined to increase/decrease the appropriate glancing angles $\theta_{1}, \theta_{2}$ and $\theta_{2, \text { tr }}$ between the positive $x$ axis and the wavevectors $\mathbf{k}_{0}^{v}, \mathbf{k}_{h}^{v}$ and $\mathbf{k}_{\mathrm{tr}}^{v}$, respectively (see Fig. 2).

Let us describe a two-dimensional rectangular lattice as shown in Fig. 3. We assume that $d$ is the distance between the atomic planes $(h k l)$, so their vertical positions are $z_{n}=n d$ ( $n$ is an integer). Then, following Punegov et al. (2014), we can choose $\Delta x=d \cot \theta_{\mathrm{B}}$ as a step size along the $x$ axis to indicate the positions $x_{m}=m \Delta x$ ( $m$ is an integer) where this beam [in a finite difference form of Takagi's equations (2)] will be partially transmitted to the next atomic plane or partially reflected. This defines a two-dimensional rectangular lattice with step sizes $d$ and $\Delta x$ along the $z$ and $x$ directions, respectively. Note that the positions of nodes $(n, m)$ (see Fig. 3) in this lattice are fixed and do not depend on either $\theta_{1}$ or $\theta_{2}$, or $\theta_{2, \mathrm{tr}}$. Now we can rewrite (2) in the finite difference form on the two-dimensional rectangular lattice shown in Fig. 3:

$$
\begin{aligned}
T_{n+1}^{m} & =t T_{n}^{m-1}+\bar{r} S_{n}^{m-1}, \\
S_{n}^{m} & =\bar{t} S_{n+1}^{m-1}+r T_{n+1}^{m-1},
\end{aligned}
$$

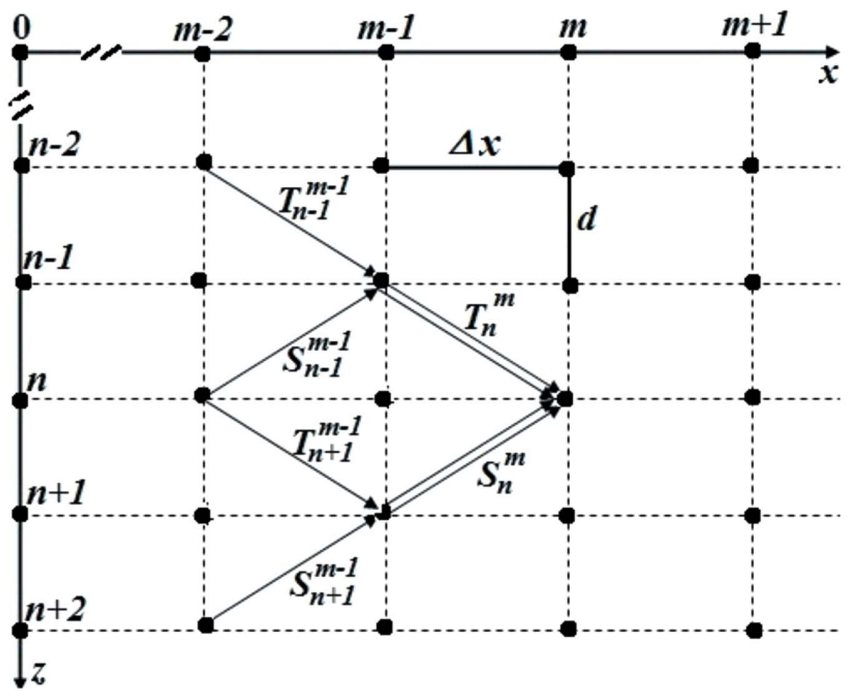

Figure 3

A schematic of dynamical diffraction on a lateral crystal, where the $(n, m)$ nodes approximately indicate where the beam will be partially transmitted to the next atomic plane or partially reflected. where $T_{n}^{m}$ and $S_{n}^{m}$ are the amplitudes of the transmitted, $E_{0}$, and reflected, $E_{h}$, waves, respectively, at the $(m ; n)$ node of a two-dimensional rectangular lattice (see Fig. 3); $t=\bar{t}=\left(1+r_{0}\right), \quad \bar{r}=r_{\bar{h}} \exp [i \mathbf{h} \cdot \mathbf{u}(\mathbf{r})], \quad r=r_{h} \exp [-i \mathbf{h} \cdot \mathbf{u}(\mathbf{r})]$, $r_{g}=i \chi_{g} \pi d /\left(\sin \theta_{\mathrm{B}} \lambda\right)(g=0, h, \bar{h})$. Equations (6) are similar to the ones we obtained previously (Punegov et al., 2014) for the Bragg geometry diffraction case using Darwin's approach. The difference is in the absence of the phase terms, which are now included in the boundary conditions [see $(4 a),(4 b),(4 c),(4 d)$ ], and in the presence of terms $\exp [ \pm i \mathbf{h} \cdot \mathbf{u}(\mathbf{r})]$ describing the deformation field. Now these equations can be employed in the case of the Bragg-Laue geometry, where an extended set of boundary conditions [see $(4 a),(4 b),(4 c),(4 d)$ ] will be used. In the Bragg geometry the diffracted wave $S^{(\mathrm{B})}$ and the transmitted wave $T^{(\mathrm{B})}$ are registered on the top surface and bottom surface, respectively. In the Laue geometry both waves, $S^{(\mathrm{L})}$ and $T^{(\mathrm{L})}$, are registered at the right vertical face of the crystal (see Fig. 2).

It should also be noted that a common multiplicative coefficient can be used to increase the step sizes $d$ and $\Delta x$. This will speed up the simulation process. For instance, if one uses $20 d$ as the vertical step size instead of $d$ and $20 \Delta x$ as the horizontal step size instead of $\Delta x$, the simulation time decreases by 400 times. However, such an increase should be implemented cautiously, to avoid artefacts.

The number of periods of the two-dimensional rectangular lattice, $M_{x}$ and $N_{z}$, along the $x$ and $z$ axes, respectively, is determined by the structure width, $L_{x}=M_{x} \Delta x$, and thickness, $L_{z}=N_{z} d$.

We can now rewrite the four boundary conditions given in $(4 a),(4 b),(4 c)$ and $(4 d)$ in a scalar form to simplify their use in recurrence relations (6):

(a) For the transmitted waves inside the crystal: at the top surface, $T_{0}^{m}$, and left vertical face, $T_{n}^{0}$, of the crystal

$$
\begin{aligned}
T_{0}^{m} & =\exp \left(i \varphi_{x, \text { in }}^{m}\right)=\exp \left[i(2 \pi / \lambda) m \Delta x\left(\cos \theta_{1}-\cos \theta_{\mathrm{B}}\right)\right], \\
T_{n}^{0} & =\exp \left(i \varphi_{z, \text { in }}^{n}\right)=\exp \left[i(2 \pi / \lambda) n d\left(\sin \theta_{1}-\sin \theta_{\mathrm{B}}\right)\right] .
\end{aligned}
$$

(b) For the diffracted waves just outside the crystal: at the top surface, $E_{h}^{v}(m \Delta x, 0)$, and right vertical face, $E_{h}^{v}\left(M_{x} \Delta x, n d\right)$, of the crystal

$$
\begin{aligned}
E_{h}^{v}(m \Delta x, 0)= & S_{0}^{m} \exp \left(i \varphi_{x, S}^{m}\right) \\
= & S_{0}^{m} \exp \left[i(2 \pi / \lambda) m \Delta x\left(\cos \theta_{\mathrm{B}}-\cos \theta_{2}\right)\right], \\
E_{h}^{v}\left(M_{x} \Delta x, n d\right)= & S_{n}^{M_{x}} \exp \left(i \varphi_{z, S}^{n}\right) \\
= & S_{n}^{M_{x}} \exp \left\{i ( 2 \pi / \lambda ) \left[M_{x} \Delta x\left(\cos \theta_{\mathrm{B}}-\cos \theta_{2}\right)\right.\right. \\
& \left.\left.-n d\left(\sin \theta_{\mathrm{B}}-\sin \theta_{2}\right)\right]\right\} .
\end{aligned}
$$

(c) For the transmitted waves just outside the crystal: at the bottom surface, $E_{\mathrm{tr}}^{v}\left(m \Delta x, N_{z} d\right)$, and right vertical face, $E_{\mathrm{tr}}^{v}\left(M_{x} \Delta x, n d\right)$, of the crystal 


$$
\begin{aligned}
E_{\mathrm{tr}}^{v}\left(m \Delta x, N_{z} d\right)= & T_{N_{z}}^{m} \exp \left(i \varphi_{x, T}^{m}\right) \\
= & T_{N_{z}}^{m} \exp \left\{i ( 2 \pi / \lambda ) \left[m \Delta x\left(\cos \theta_{\mathrm{B}}-\cos \theta_{2, \mathrm{tr}}\right)\right.\right. \\
& \left.\left.+N_{z} d\left(\sin \theta_{\mathrm{B}}-\sin \theta_{2, \mathrm{tr}}\right)\right]\right\}, \\
E_{\mathrm{tr}}^{v}\left(M_{x} \Delta x, n d\right)= & T_{n}^{M_{x}} \exp \left(i \varphi_{z, T}^{n}\right) \\
= & T_{n}^{M_{x}} \exp \left\{i ( 2 \pi / \lambda ) \left[M_{x} \Delta x\left(\cos \theta_{\mathrm{B}}-\cos \theta_{2, \mathrm{tr}}\right)\right.\right. \\
& \left.\left.+n d\left(\sin \theta_{\mathrm{B}}-\sin \theta_{2, \mathrm{tr}}\right)\right]\right\} .
\end{aligned}
$$

(d) For the diffracted waves inside the crystal: at the left vertical face, $S_{n}^{0}$, and bottom surface, $S_{N_{z}}^{m}$, of the crystal (to indicate the absence of the incoming diffracted wave from this particular direction)

$$
S_{n}^{0}=S_{N_{z}}^{m}=0 .
$$

The simulation procedure based on (6) is described in detail by Punegov et al. (2014). The difference is only in the requirement to use the boundary conditions at all external surfaces of the lateral crystal.

The amplitude reflection $S\left(q_{x}, q_{z}\right)$ and transmission $T\left(q_{\mathrm{tr}, x}, q_{\mathrm{tr}, z}\right)$ coefficients of the lateral plane-parallel crystalline structure are the following sums:

$$
\begin{gathered}
S\left(q_{x}, q_{z}\right)=S^{(\mathrm{B})}\left(\theta_{1}, \theta_{2}\right)+S^{(\mathrm{L})}\left(\theta_{1}, \theta_{2}\right), \\
T\left(q_{\mathrm{tr}, x}, q_{\mathrm{tr}, z}\right)=T^{(\mathrm{B})}\left(\theta_{1}, \theta_{2, \mathrm{tr}}\right)+T^{(\mathrm{L})}\left(\theta_{1}, \theta_{2, \mathrm{tr}}\right) .
\end{gathered}
$$

Here we distinguish the coefficients relating to the local Bragg geometry diffraction (see Fig. 2),

$$
\begin{aligned}
S^{(\mathrm{B})}\left(\theta_{1}, \theta_{2}\right) & =\sum_{m=0}^{M_{x}} S_{0}^{m} \exp \left(i \varphi_{x, S}^{m}\right), \\
T^{(\mathrm{B})}\left(\theta_{1}, \theta_{2, \mathrm{tr}}\right) & =\sum_{m=0}^{M_{x}} T_{N_{z}}^{m} \exp \left(i \varphi_{x, T}^{m}\right),
\end{aligned}
$$

and the coefficients relating to the local Laue geometry diffraction (see Fig. 2),

$$
\begin{aligned}
S^{(\mathrm{L})}\left(\theta_{1}, \theta_{2}\right) & =\sum_{n=1}^{N_{z}} S_{n}^{M_{x}} \exp \left(i \varphi_{z, S}^{n}\right), \\
T^{(\mathrm{L})}\left(\theta_{1}, \theta_{2, \mathrm{tr}}\right) & =\sum_{n=1}^{N_{z}} T_{n}^{M_{x}} \exp \left(i \varphi_{z, T}^{n}\right) .
\end{aligned}
$$

The connection between the angular parameters $\theta_{1 ; 2}=\theta_{\mathrm{B}}+\Delta \theta_{1 ; 2}$ and the projections of the reciprocal space vectors $q_{x}, q_{z}$ is as follows ( $c f$. Iida \& Kohra, 1979):

$$
\begin{aligned}
& q_{x}=k \sin \theta_{\mathrm{B}}\left(\Delta \theta_{1}-\Delta \theta_{2}\right), \\
& q_{z}=-k \cos \theta_{\mathrm{B}}\left(\Delta \theta_{1}+\Delta \theta_{2}\right) .
\end{aligned}
$$

The components of the newly introduced vector $\mathbf{q}_{\mathrm{tr}}$ depend on $\theta_{1 ; 2, \mathrm{tr}}=\theta_{\mathrm{B}}+\Delta \theta_{1 ; 2, \mathrm{tr}}$ :

$$
\begin{aligned}
& q_{\mathrm{tr}, x}=k \sin \theta_{\mathrm{B}}\left(\Delta \theta_{1}-\Delta \theta_{2, \mathrm{tr}}\right), \\
& q_{\mathrm{tr}, z}=k \cos \theta_{\mathrm{B}}\left(\Delta \theta_{2, \mathrm{tr}}-\Delta \theta_{1}\right) .
\end{aligned}
$$
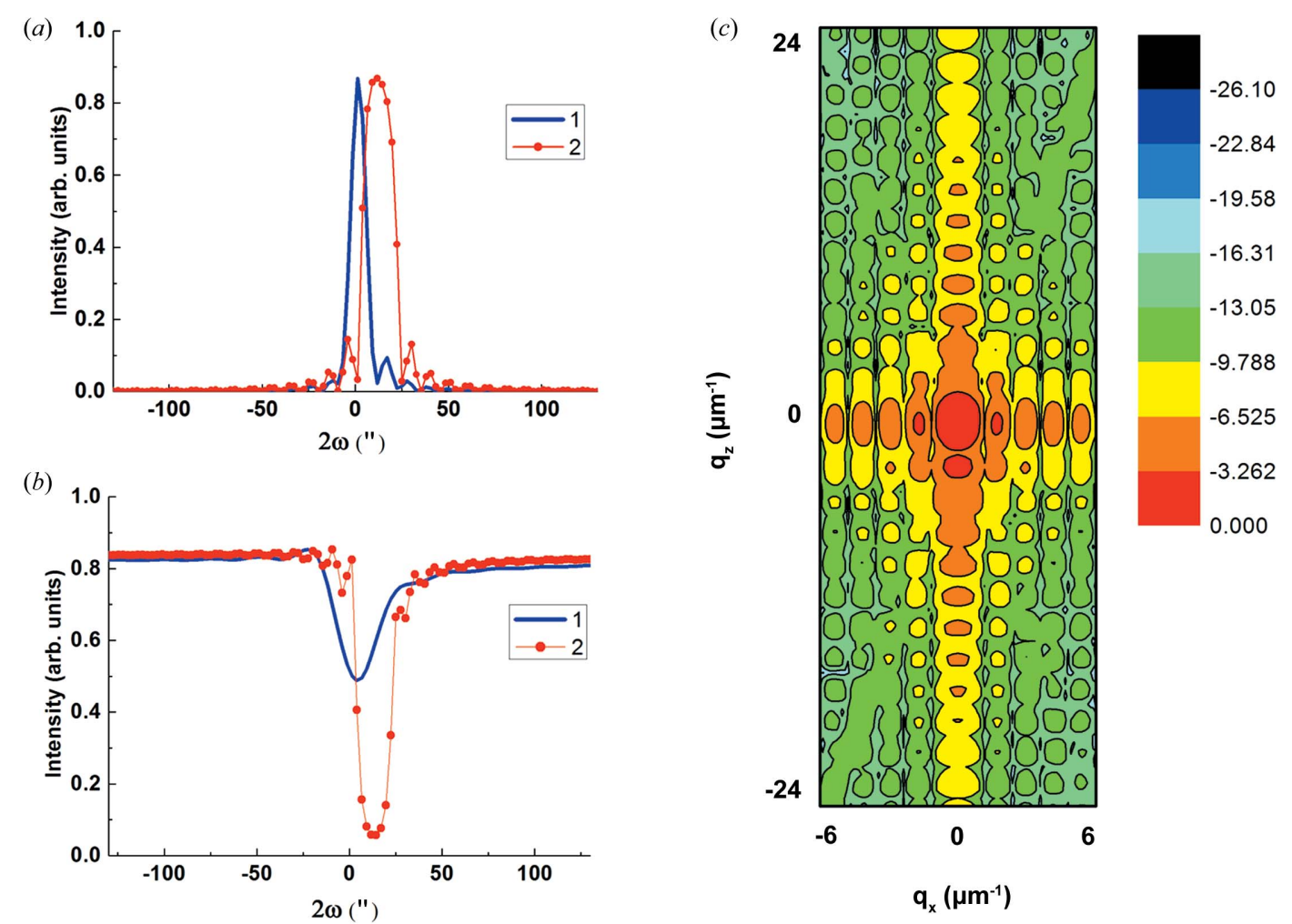

Figure 4

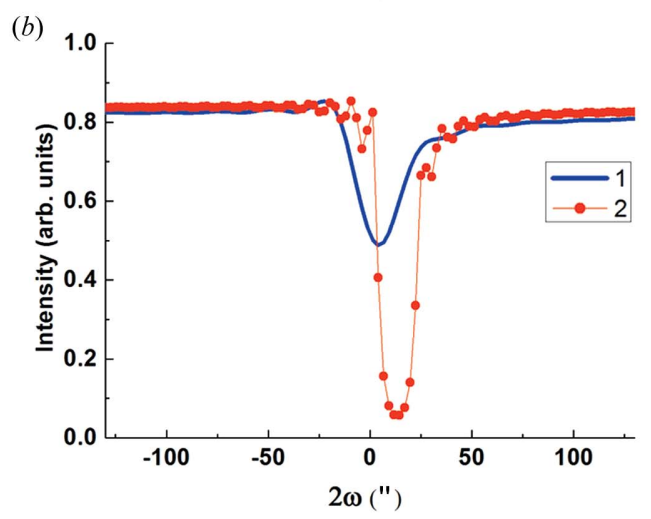

(a) The reflection rocking curve, $(b)$ the transmission rocking curve and (c) the normalized diffracted RSM for a lateral crystal with thickness $L_{z}=3 \mu \mathrm{m}$ and width $L_{x}=5 \mu \mathrm{m}$. Red lines (2) in (a) and (b) show simulations for a crystal with width $L_{x}=\infty$. The RSM is shown in logarithmic scale. 

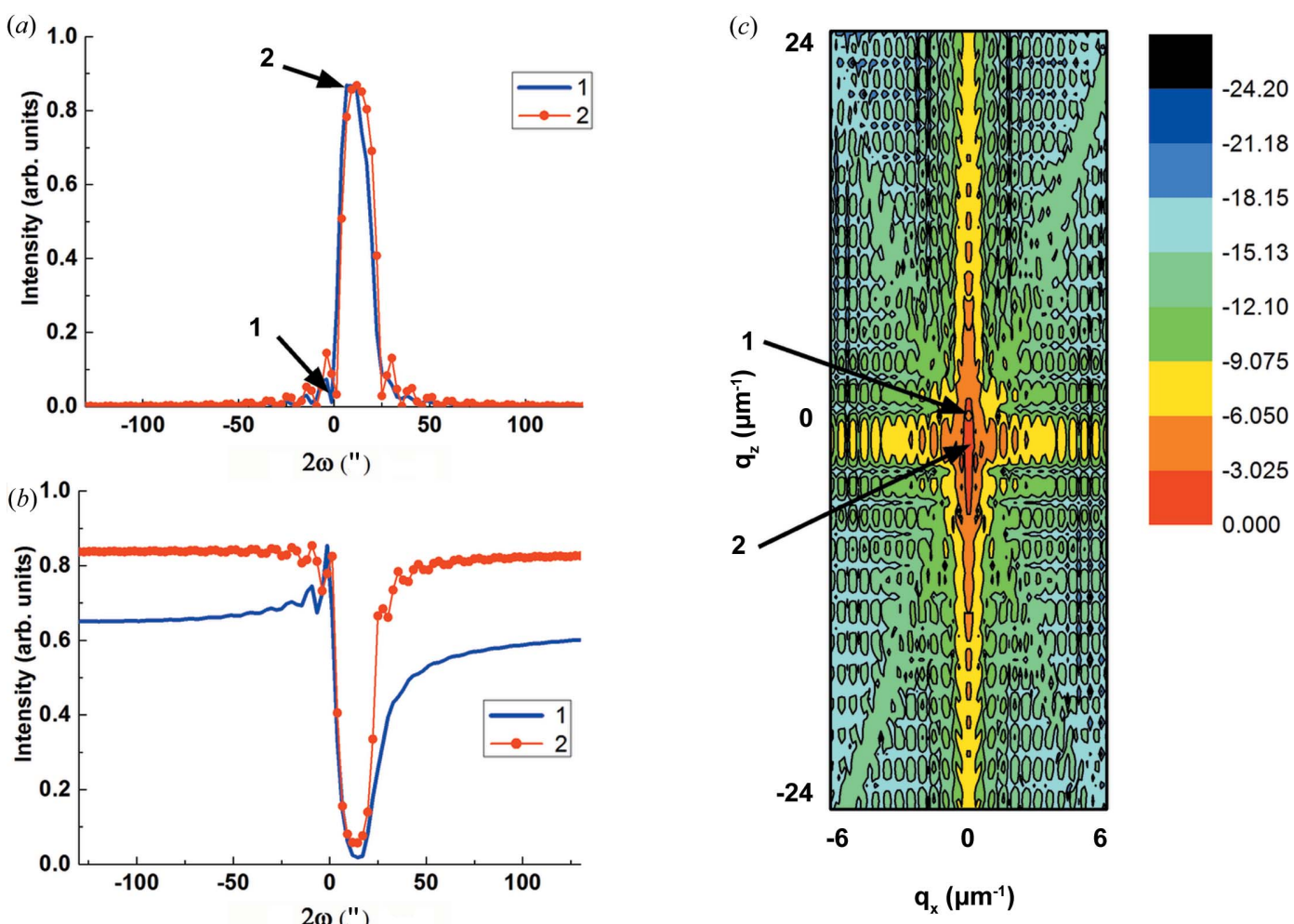

Figure 5

(a) The reflection rocking curve, $(b)$ the transmission rocking curve and $(c)$ the normalized diffracted RSM for a lateral crystal with thickness $L_{z}=3 \mu \mathrm{m}$ and width $L_{x}=37.4 \mu \mathrm{m}$. Red lines (2) in $(a)$ and $(b)$ show simulations for a crystal with width $L_{x}=\infty$. Points 1 and 2 correspond to the minimum and maximum diffracted intensity, respectively, near the node (111) in reciprocal space. The RSM is shown in logarithmic scale.
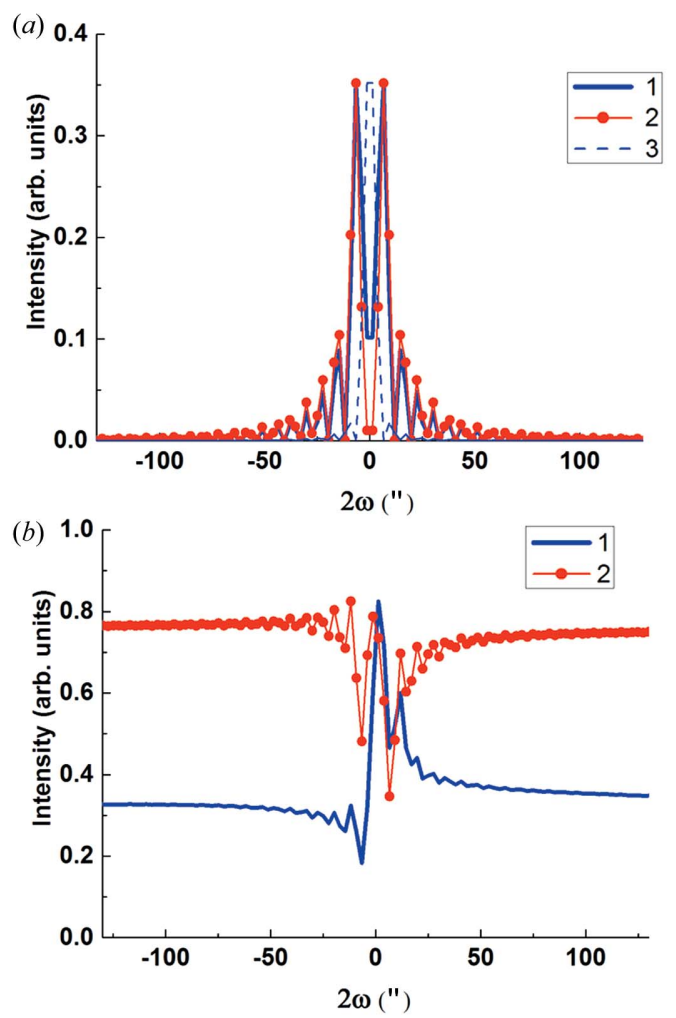

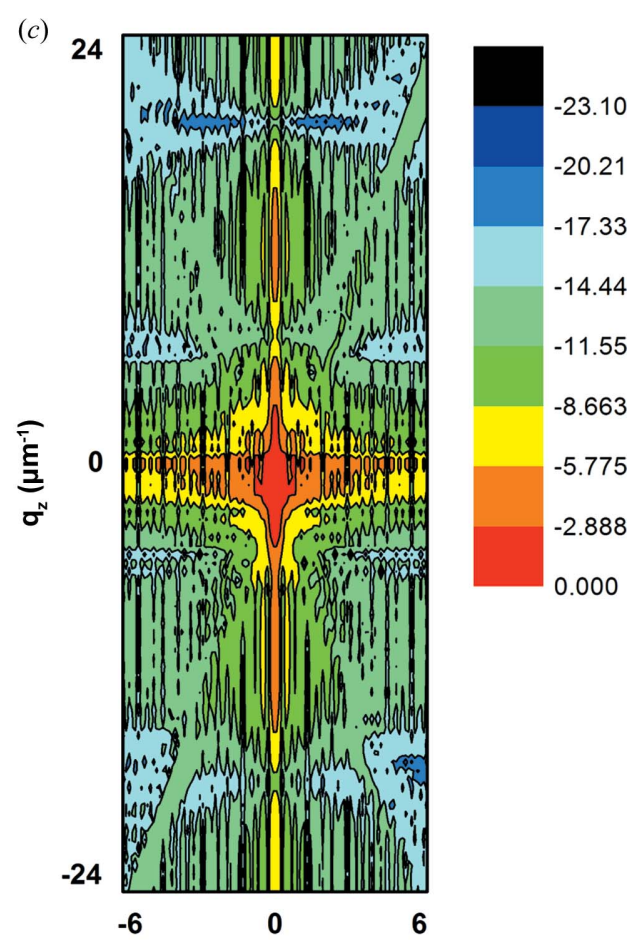

$q_{x}\left(\mu m^{-1}\right)$

Figure 6

(a) The reflection rocking curve, $(b)$ the transmission rocking curve and $(c)$ the normalized diffracted RSM for a lateral crystal with thickness $L_{z}=13 \mu \mathrm{m}$ and width $L_{x}=18.7 \mu \mathrm{m}$. Red lines (2) in $(a)$ and $(b)$ show simulations for a crystal with thickness $L_{z}=\infty$. The blue dashed line (3) in $(a)$ shows simulations using kinematical diffraction theory. The RSM is shown in logarithmic scale. 
In the so-called $\omega-2 \omega$ (or $\theta-2 \theta$ ) scanning mode there is the following connection between the angular deviations for the registered diffracted beam in the Bragg geometry: $\Delta \theta_{1}=$ $\Delta \theta_{2}=\omega$. Then $q_{x}=0$ and $q_{z}=-k \cos \theta_{\mathrm{B}}\left(2 \Delta \theta_{1}\right)=$ $-2 k \cos \theta_{\mathrm{B}} \omega$. This scanning mode condition $\Delta \theta_{1}=\Delta \theta_{2}=\omega$ also satisfies the so-called lateral (for the top surface of the lateral crystal) diffraction condition [see e.g. ch. 4 in Pietsch et al. (2004)] and corresponds to a crystal truncation rod (CTR) scan along the $q_{z}$ direction.

The CTR scan in the Laue geometry is performed along the $q_{x}$ direction. Then, for the registered diffracted beam $\Delta \theta_{1}=$ $-\Delta \theta_{2}=\omega, q_{x}=k \sin \theta_{\mathrm{B}}\left(2 \Delta \theta_{1}\right)=2 k \omega \sin \theta_{\mathrm{B}}$ and $q_{z}=0$.

To follow the direction of the transmitted wave for both the Laue and Bragg geometries, the condition $\Delta \theta_{2, \mathrm{tr}}=\Delta \theta_{1}=\omega$ must be satisfied to enable the comparison with the traditional dynamical diffraction approaches (16) and (17). This makes $q_{\mathrm{tr}, x}=q_{\mathrm{tr}, z}=0$.

The above-mentioned conditions $\left(\Delta \theta_{1}=\Delta \theta_{2}=\omega\right.$ for the $q_{z}$ scan, $\Delta \theta_{1}=-\Delta \theta_{2}=\omega$ for the $q_{x}$ scan and $\Delta \theta_{2, \text { tr }}=$ $\Delta \theta_{1}=\omega$ for the transmitted beam) will be used in $\$ 3$ to compare the simulations done using (6), (8) and (9) and the traditional dynamical diffraction approaches (13), (14), (16) and (17).

\section{Numerical modelling}

The numerical modelling of RSMs and directional scans in reciprocal space for the transmitted and diffracted waves, in
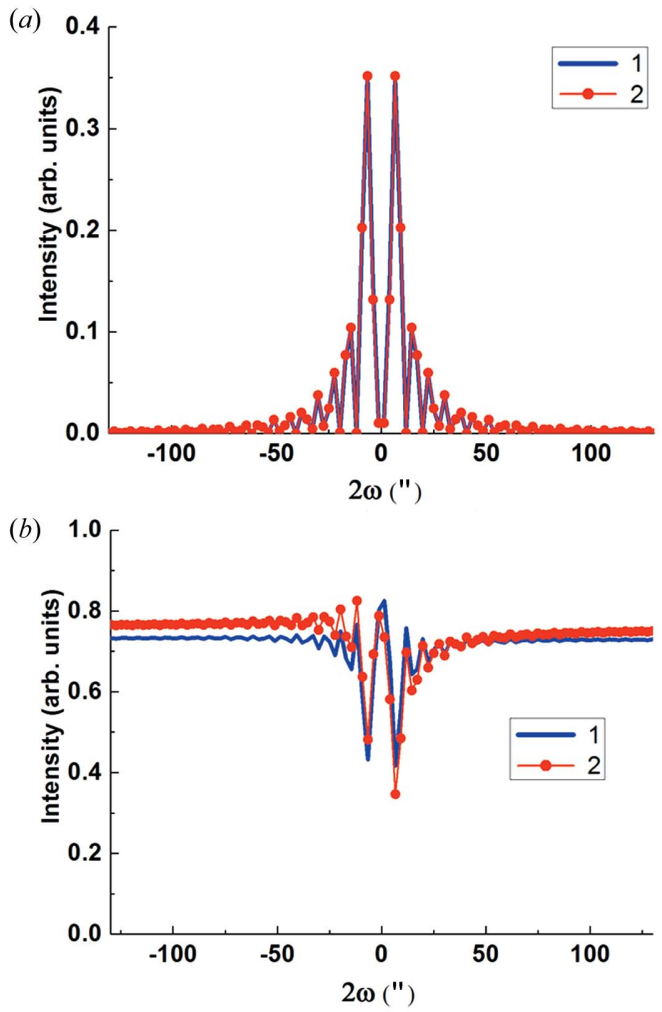

the case of the lateral crystal, is performed using (6), (8) and (9). For comparison, the modelling of Bragg or Laue diffraction for a crystal having an infinite extent in one direction was done using (13) and (14) or (16) and (17), respectively. In our simulations we use $\mathrm{Cu} K \alpha_{1}$ radiation (the wavelength is $0.154056 \mathrm{~nm}$ ) for the (111) reflection of an $\mathrm{Si}$ crystalline structure. The appropriate Bragg angle is $\theta_{\mathrm{B}}=14.22^{\circ}$. The primary extinction length (Authier, 2001) for the $\mathrm{Si}(111)$ Bragg diffraction case is $1.506 \mu \mathrm{m}$ (Stepanov \& Forrest, 2008); the Pendellösung distance for the Laue diffraction case is $18.67 \mu \mathrm{m}$ (Stepanov \& Forrest, 2008).

In Figs. 4-7 the rocking-curve simulations for a lateral crystal, based on (8) and (9), are labelled 1, and the Bragg [(13) and (14)] and Laue [(16) and (17)] diffraction case simulations for a crystal having an infinite extent in one direction are labelled 2. In an effort to apply proper normalization, all simulated reflected/transmitted-wave intensities for a lateral crystal were normalized on the maximum intensity of the rocking curves for a plane-parallel crystal [(13), (14), (16) and (17)] of the same thickness. We can say for a lateral crystal that the Bragg-Laue diffraction case applies if $L_{x}>L_{z}$. Then, if $L_{x}<L_{z}$, the Laue-Bragg diffraction case applies. All RSMs in Figs. 4-7 are shown in a logarithmic scale. A step size of 0.178 for intensity maps was used in Figs. 8 and 9.

\subsection{Bragg-Laue diffraction}

First we consider the case $L_{x}>L_{z}$, where the major effect is caused by Bragg diffraction and Laue diffraction plays a minor

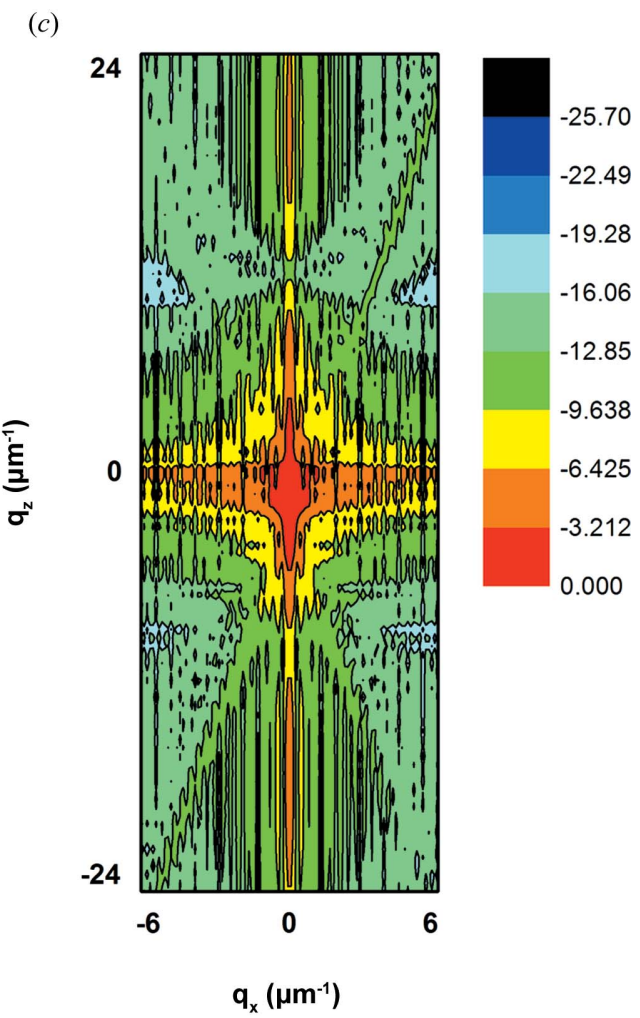

Figure 7

(a) The reflection rocking curve, $(b)$ the transmission rocking curve and (c) the normalized diffracted RSM for a lateral crystal with thickness $L_{z}=63 \mu \mathrm{m}$ and width $L_{x}=18.7 \mu \mathrm{m}$. Red lines (2) in $(a)$ and $(b)$ show simulations for a crystal with thickness $L_{z}=\infty$. The RSM is shown in logarithmic scale. 
role. For the same crystal thickness, $L_{z}$, of $3 \mu \mathrm{m}$, which is twice the primary extinction length, we compare the simulation results for two different crystal widths, namely a small lateral width $\left(L_{x}=5 \mu \mathrm{m}\right)$ and a large lateral width $\left(L_{x}=37.4 \mu \mathrm{m}\right)$

Fig. 4 shows simulations for the reflection rocking curve (Fig. 4a, blue line 1), transmission rocking curve (Fig. $4 b$, blue line 1) and diffracted RSM for a crystal width $L_{x}$ of $5 \mu \mathrm{m}$. For comparison, also shown are (red lines labelled 2) the reflection (Fig. $4 a$ ) and transmission (Fig. $4 b$ ) rocking curves for a crystal having an infinite extent in the $x$ direction. Despite the crystal thickness, $L_{z}$, still being the same, the simulation curves for a lateral crystal (blue lines) and a plane-parallel crystal (red lines) are markedly distinguished. For such a 'narrow' lateral crystal, the dynamical interaction between the transmitted and diffracted wave cannot properly occur, and the rocking curves and RSM demonstrate the semi-dynamical character of such an interaction.

For the case of a 'wide' lateral crystal with width $L_{x}=$ $37.4 \mu \mathrm{m}$ (see Figs. $5 a$ and $5 b$ ) the difference between rocking curves calculated for a lateral crystal $\left(L_{x}=37.4 \mu \mathrm{m}\right)$ and a plane-parallel crystal $\left(L_{x}=\infty\right)$ is markedly smaller. The existing difference can be explained by the redirection of intensity in the Laue diffraction channel. Fig. 5(c) shows the diffracted intensity RSM for a 'wide' lateral crystal with width $L_{x}=37.4 \mu \mathrm{m}$ near the node (111) in reciprocal space, which corresponds to the origin of Fig. 5(c). Points 1 and 2, shown in

(a)

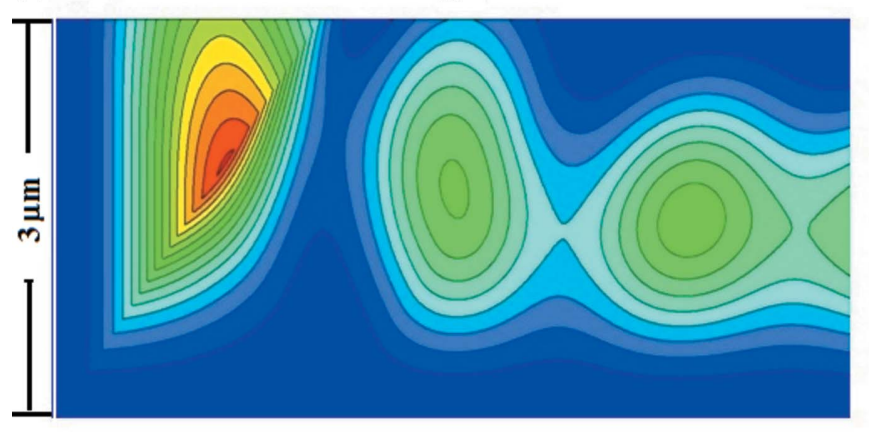

(b)

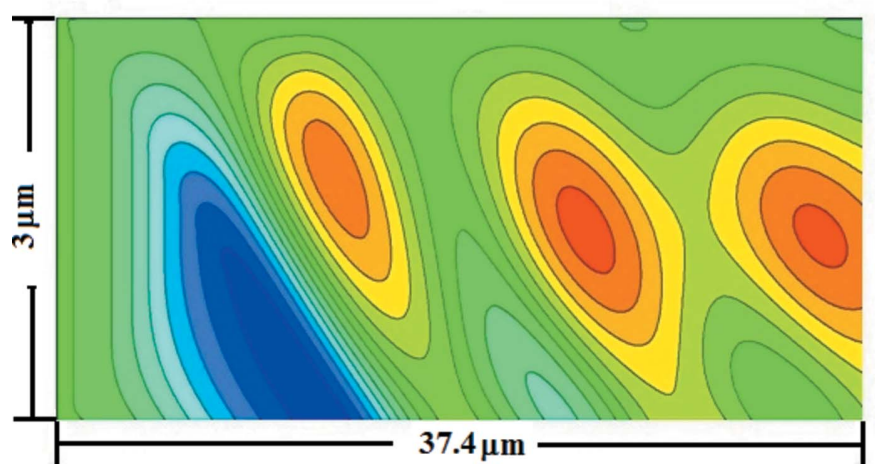

Figure 8

(a) The reflection and (b) the transmission X-ray intensity distribution maps (in linear scale with a step size of 0.05 for intensity) at $\omega=0$ inside a crystal having width $L_{x}=37.4 \mu \mathrm{m}$ and thickness $L_{z}=3 \mu \mathrm{m}$. The red and blue colours correspond to 1 (maximum) and 0.05 (minimum), respectively.
Fig. 5, correspond to the minimum and maximum diffracted intensity, respectively, near the node (111) in reciprocal space.

\subsection{Laue-Bragg diffraction}

Now we consider the case of Laue-Bragg diffraction, where the major role is played by Laue diffraction. To analyse this case, one needs to choose $L_{x}<L_{z}$ for the lateral crystal. Our simulations are done for two lateral crystals having the same width $L_{x}=18.7 \mu \mathrm{m}$, which coincides with the Pendellösung distance, and different thicknesses, $L_{z}$, namely 13 and $63 \mu \mathrm{m}$.

Fig. 6 shows the simulations for the reflection rocking curve (Fig. 6a, blue line 1), transmission rocking curve (Fig. $6 b$, blue line 1) and diffracted RSM (Fig. 6c) for a crystal thickness $L_{z}$ of $13 \mu \mathrm{m}$. The red line in Figs. 6(a) and 6(b) shows the simulations [based on (16) and (17)] for a crystal having an infinite thickness, i.e. $L_{z}=\infty$. There is a substantial difference between these simulation results. However, both demonstrate typical characteristic features of Laue diffraction. The blue dashed line in Fig. 6(a) shows the kinematical diffraction simulation [based on equation 3.9 of Authier (2001)]. This demonstrates that the kinematical theory cannot correctly describe the dynamical character of interaction between the transmitted and diffracted wave in the Laue diffraction case.

For the second crystal, having a thickness $L_{z}$ of $63 \mu \mathrm{m}$, the simulation results (see Fig. 7) are very similar to those [based on (16) and (17)] for a crystal with thickness $L_{z}=\infty$. The

(a)

$\mathrm{R}$

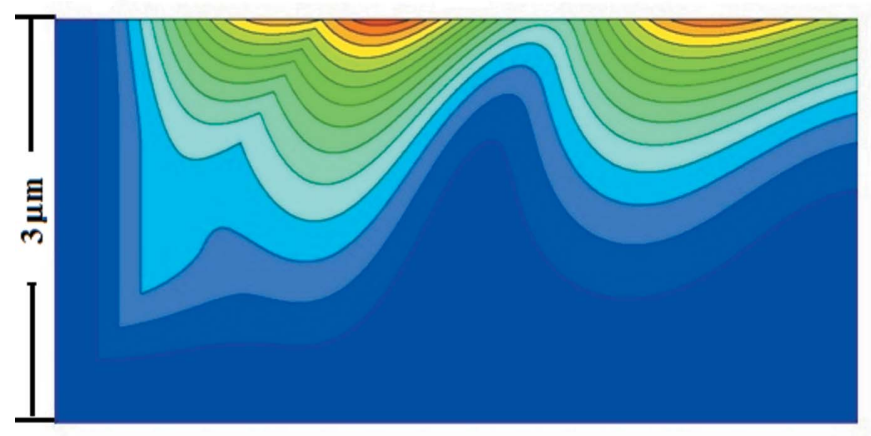

(b)

$\mathrm{T}$

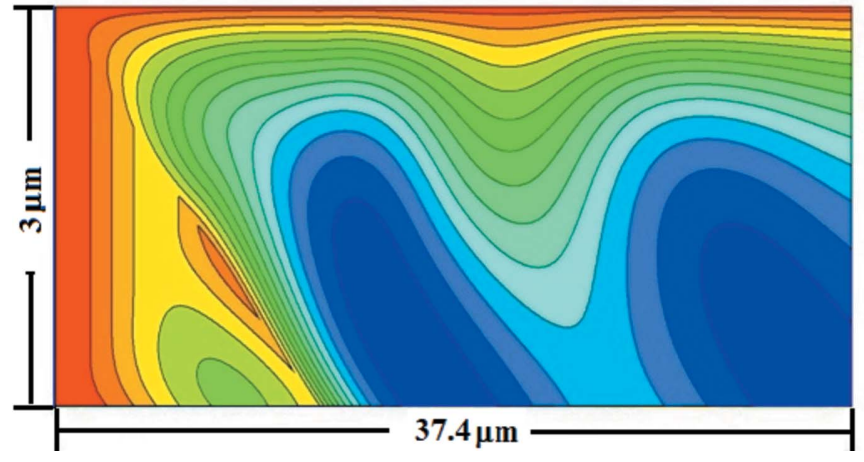

Figure 9

(a) The reflection and (b) the transmission X-ray intensity distribution maps (in linear scale with a step size of 0.05 for intensity) at $\omega=5^{\prime \prime}$ inside a crystal having width $L_{x}=37.4 \mu \mathrm{m}$ and thickness $L_{z}=3 \mu \mathrm{m}$. The red and blue colours correspond to 1 (maximum) and 0.05 (minimum), respectively. 
observed small difference between the red $\left(L_{z}=\infty\right)$ and blue $\left(L_{z}=63 \mu \mathrm{m}\right)$ lines can be explained by the Bragg diffraction channel, where a part of the intensity escapes through the top and bottom surfaces of the lateral crystal.

\subsection{X-ray wavefields inside the lateral crystal}

Figs. 8 and 9 show the reflection and transmission X-ray intensity distribution maps at points 1 and 2 shown in Fig. 5(a), respectively, inside a crystal having width $L_{x}=37.4 \mu \mathrm{m}$ and thickness $L_{z}=3 \mu \mathrm{m}$. The maps are given using a linear scale with a step size of 0.05 for intensity. Red and blue colours correspond to 1 (maximum) and 0.05 (minimum) intensity, respectively.

The crystal width corresponds to two of the Pendellösung distances in the Laue geometry. The diffraction and transmission rocking curves as well as RSMs for this crystal are shown in Fig. 5. In particular, the angular positions $\omega=0$ and $\omega=5^{\prime \prime}$ correspond to points $1\left(q_{z}=0\right)$ and $2\left(q_{z}=-2 \mu \mathrm{m}^{-1}\right)$, respectively. It should be noted that in this particular case Bragg diffraction plays the major role.

First we consider the case when $\omega=0$. As the Darwin curve centre is shifted owing to refraction by about $7^{\prime \prime}$, the Bragg diffraction intensity (see Fig. 8a) at this angular position is small (see also Fig. 5). However, in the Laue geometry there is no such refraction shift; therefore this angular position is expected to have a high intensity for the Laue diffraction case (see Fig. $8 b$ ) in a lateral crystal having thickness $L_{z}=3 \mu \mathrm{m}$. At the same time, this Laue type of diffraction is relatively weak since the left vertical face of the lateral crystal is only $3 \mu \mathrm{m}$. Nevertheless, we observe the Pendellösung effects in the form of ellipses in both the diffracted (Fig. 8a) and transmitted (Fig. 8b) wavefields.

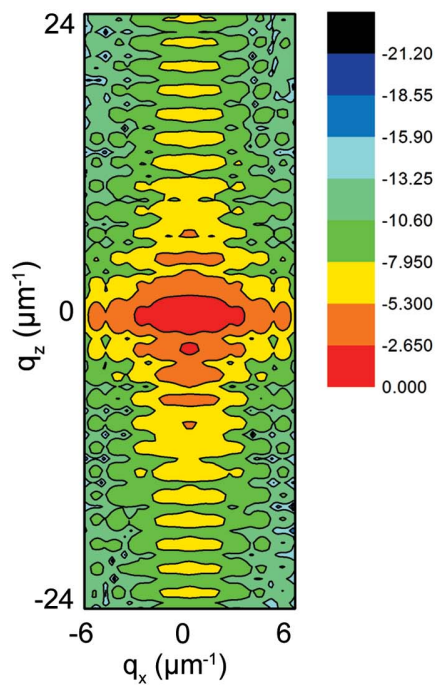

(a)

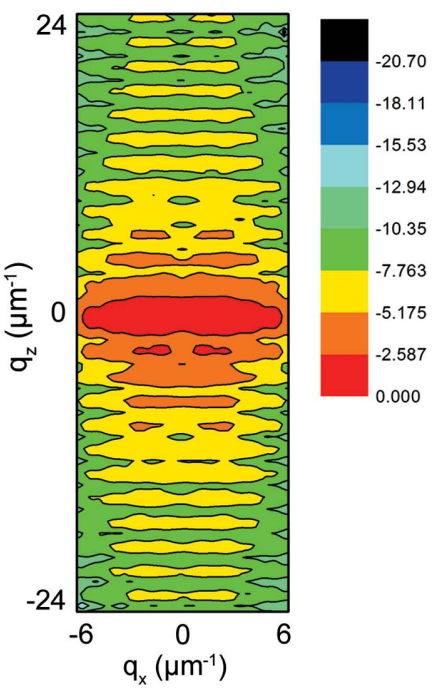

(b)
Figure 10

RSMs for a deformed lateral crystal with thickness $L_{z}=3 \mu \mathrm{m}$ and width $L_{x}=5 \mu \mathrm{m}$. The maximum phase shift, $\varphi(\mathbf{r})$, caused by the deformation is (a) $\pi$ and (b) $2 \pi$. The RSMs are shown in logarithmic scale.
When $\omega=5^{\prime \prime}$ (or $q_{z}=-2 \mu \mathrm{m}^{-1}$ ) (see Fig. 9), which corresponds to point 2 in Fig. 5, we observe the maximum intensity in the Bragg diffracted wavefield at the top surface of the lateral crystal (see Fig. 9a). One can see two areas of such strong intensity near the top surface. Such inhomogeneity is caused by the Laue diffraction effects at the left and right vertical faces of the lateral crystal.

The maximum value of transmitted intensity at the top left corner of Fig. $9(b)$ is caused by the boundary conditions: in particular, the X-ray wave is incident on the left vertical face and top surface of the lateral crystal. The further propagation inside the crystal diminishes the transmitted-wave intensity owing to the primary extinction effects caused by interference. Therewith its energy is transferred into the diffracted wave.

\subsection{Effect of the deformation field}

The approach presented in $\S 2$ allows one to simulate dynamical diffraction RSMs for a deformed lateral crystal. To illustrate this ability we use a model of the deformation field reported by Cha et al. (2010). This parabolic displacement may be associated (Cha et al., 2010) with the attachment of the lateral crystal to a substrate and can be approximated as $\exp [i \mathbf{h} \cdot \mathbf{u}(\mathbf{r})]=\exp [i \varphi(\mathbf{r})]=\exp \left[i \gamma\left(x-L_{x} / 2\right)^{2}\right]$, where $\gamma$ is a constant inversely proportional to the radius of curvature and $\varphi(\mathbf{r})$ is the phase shift caused by the deformation field. In our simulations we have chosen the values of the coefficient $\gamma$ to have a maximum of the phase shift, $\varphi(\mathbf{r})$, of either $\pi$ or $2 \pi$ radians at the edges of the lateral crystal. Such a phase shift is the so-called strong phase limit, which is extremely likely to occur in practice (Cha et al., 2010). However, the supportbased phasing method, used in the iterative reconstruction procedure reported by Cha et al. (2010), was not successful for such large phase shifts.

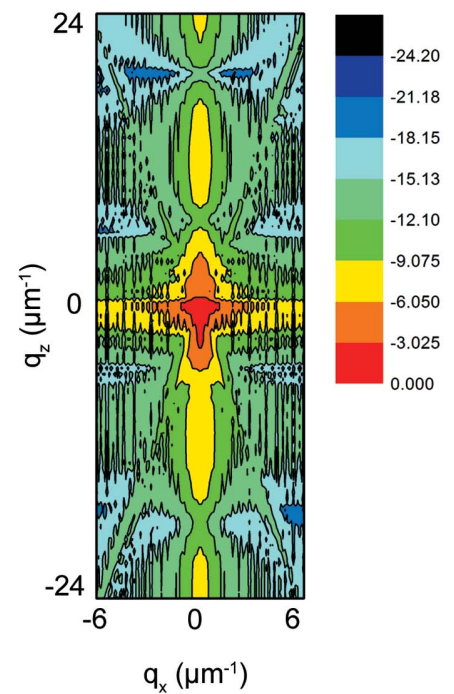

(a)

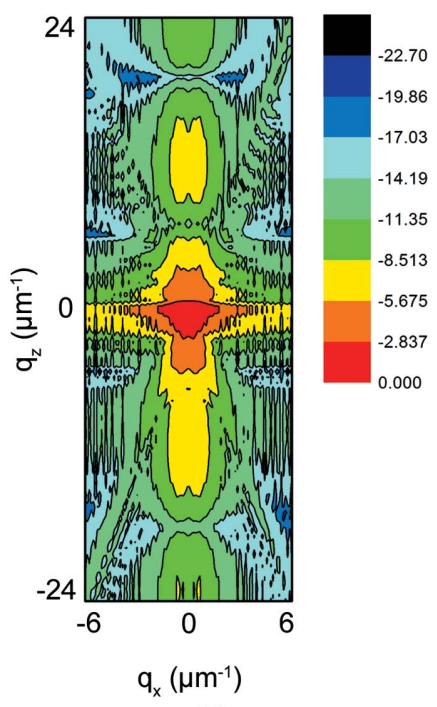

(b)
Figure 11

RSMs for a deformed lateral crystal with thickness $L_{z}=13 \mu \mathrm{m}$ and width $L_{x}=18.7 \mu \mathrm{m}$. The maximum phase shift, $\varphi(\mathbf{r})$, caused by the deformation is $(a) \pi$ and $(b) 2 \pi$. The RSMs are shown in logarithmic scale. 

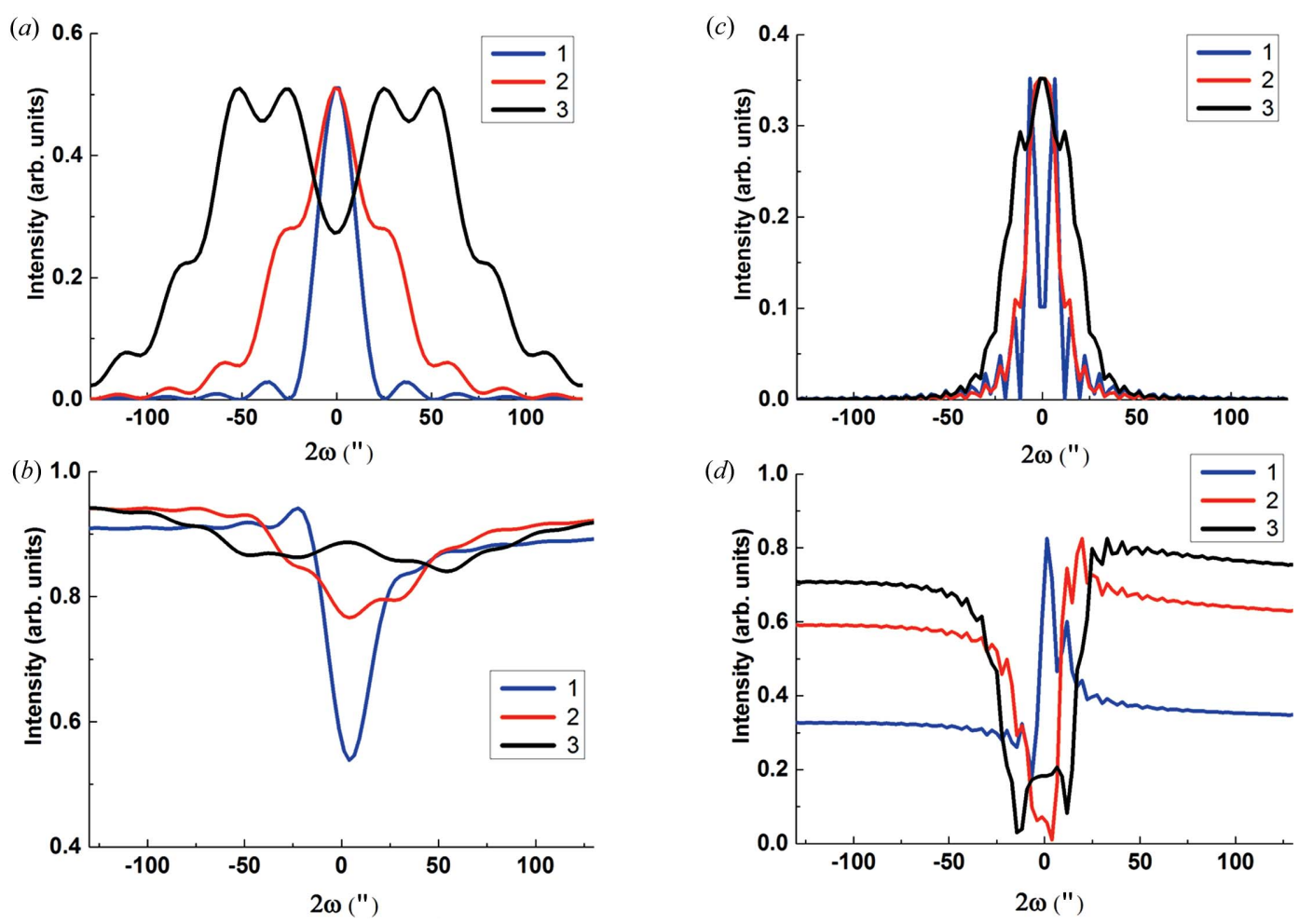

Figure 12

(a) The reflection rocking curve (a $q_{x}$ scan) and (b) the transmission rocking curve for a lateral crystal with thickness $L_{z}=3 \mu \mathrm{m}$ and width $L_{x}=5 \mu \mathrm{m}$. (c) The reflection rocking curve (a $q_{x}$ scan) and $(d)$ the transmission rocking curve for a lateral crystal with thickness $L_{z}=13 \mu \mathrm{m}$ and width $L_{x}=18.7 \mu \mathrm{m}$. The maximum phase shift, $\varphi(\mathbf{r})$, caused by the deformation is either zero (blue lines 1 ) or $\pi$ (red lines 2 ), or $2 \pi$ (black lines 3 ).

We applied this deformation field to two of the lateral crystals considered in this paper, namely the lateral crystal having thickness, $L_{z}$, of $3 \mu \mathrm{m}$ and lateral width, $L_{x}$, of $5 \mu \mathrm{m}$ (see Fig. 10 and Figs. $12 a$ and $12 b$ ) and the lateral crystal having thickness, $L_{z}$, of $13 \mu \mathrm{m}$ and lateral width, $L_{x}$, of $18.7 \mu \mathrm{m}$ (see Fig. 11 and Figs. 12c and 12d).

The simulated RSMs (Figs. 10 and 11) demonstrate broadening in the $q_{x}$ direction in comparison with the RSMs from non-deformed crystals (Figs. $4 c$ and $6 c$ ). The deformation producing the maximum phase shift, $\varphi(\mathbf{r})$, of $2 \pi$ radians causes splitting of the diffraction patterns (see Figs. $10 b$ and $11 b$ ).

Fig. 12 shows the $q_{x}$ scans (with $q_{z}=0$ ) for the diffracted intensity (Figs. $12 a$ and $12 c$ ) and the appropriate transmission rocking curves (Figs. $12 b$ and $12 d$ ). The $q_{z}$ scans do not demonstrate sensitivity to this deformation field depending on the $x$ coordinate only. These $q_{x}$ scans allow one to see the fine structure of the intensity distribution shown in RSMs (Figs. 10 and 11). In Fig. 12 the maximum phase shift, $\varphi(\mathbf{r})$, caused by the deformation is either zero (blue lines 1 ) or $\pi$ (red lines 2 ), or $2 \pi$ (black lines 3 ).

\section{Conclusions}

We have demonstrated how the dynamical theory approach for lateral crystals, first implemented in the case of nondeformed crystals for Bragg geometry (Punegov et al., 2014) using Darwin's dynamical theory, can be extended to the case of deformed crystals in mixed Bragg-Laue geometries using Takagi's equations. This approach allows calculations of RSMs in both the directions of the transmitted and diffracted waves for rectangular-shaped crystals of arbitrary sizes. This makes easier the solution of X-ray diffraction inverse problems based on minimization of the discrepancy between the simulated and experimental data (Pavlov et al., 1995; Punegov et al., 1996; Kirste et al., 2005; Vartanyants \& Yefanov, 2015) in highresolution X-ray diffractometry and CDI. The approach can be potentially extended to the three-dimensional case in both the Fourier space and real space.

\section{APPENDIX $A$}

Here we provide a short review of some classical results obtained for symmetrical Bragg and Laue X-ray diffraction on plane-parallel non-deformed crystals of a finite thickness. These results are used to simulate rocking curves in the Bragg and Laue geometries in the main part of this paper. Here we assume that the incident X-ray wave is a plane wave of unit intensity and infinite extent. Also in this paper we consider only a symmetrical coplanar diffraction case for $\sigma$ polarization.

Let us start with Takagi's equations (Takagi, 1969) describing symmetrical Bragg diffraction by an ideal crystal having thickness $L_{z}$ (see Fig. 1a). Here the angle between the wavevector of the incident plane wave and the top crystal surface is $\theta_{1}=\theta_{\mathrm{B}}+\omega$, where $\omega=\theta_{1}-\theta_{\mathrm{B}}$ is the angular deviation from the Bragg angle $\theta_{\mathrm{B}}$ for the family of reflecting atomic planes $(h k l)$. 


$$
\left\{\begin{array}{l}
\frac{\partial}{\partial z} E_{0}\left(\eta_{z} ; z\right)=i a_{0}^{(\mathrm{B})} E_{0}\left(\eta_{z} ; z\right)+i a_{\bar{h}}^{(\mathrm{B})} E_{h}\left(\eta_{z} ; z\right) \\
-\frac{\partial}{\partial z} E_{h}\left(\eta_{z} ; z\right)=i\left[a_{0}^{(\mathrm{B})}+\eta_{z}\right] E_{h}\left(\eta_{z} ; z\right)+i a_{h}^{(\mathrm{B})} E_{0}\left(\eta_{z} ; z\right)
\end{array}\right.
$$

where $E_{0, h}\left(\eta_{z} ; z\right)$ are the amplitudes of the transmitted and diffracted waves, respectively, $a_{0}^{(\mathrm{B})}=\pi \chi_{0} /\left(\lambda\left|\sin \theta_{\mathrm{B}}\right|\right), a_{h,-h}^{(\mathrm{B})}=$ $C \pi \chi_{h,-h} /\left(\lambda\left|\sin \theta_{\mathrm{B}}\right|\right), \eta_{z}=2 k \cos \left(\theta_{\mathrm{B}}\right) \omega$ is the angular parameter used in the so-called $\omega-2 \omega$ scanning mode (the $q_{z}$ scan in reciprocal space for the diffracted wave), $\lambda$ is the X-ray wavelength in vacuum, $k=2 \pi / \lambda, C$ is the polarization factor ( $C=1$ for the $\sigma$-polarization case considered in this paper), $\chi_{g}=-r_{0} \lambda^{2} F_{g} /\left(\pi V_{\mathrm{c}}\right)$ are the Fourier coefficients of dielectric susceptibility (polarizability) where $g=0, h, \bar{h}, F_{g}$ is the structure factor, $V_{\mathrm{c}}$ is the volume of the elementary unit cell, $r_{0}=e^{2} /\left(m c^{2}\right)$ is the classical electron radius, $c$ is the speed of light in vacuum, and $e$ and $m$ are electron charge and electron mass, respectively.

As the crystal and the incident wave are of infinite extent in the $x$ direction, the amplitudes of both the transmitted and diffracted waves depend only on the $z$ coordinate. We consider the case when the angular deviation $\omega$ is small.

Using the following boundary conditions for the incident $E_{0}\left(\eta_{z} ; z=0\right)=T=1$ and the reflected $E_{h}\left(\eta_{z} ; z=L_{z}\right)=0$ waves as well as for the amplitude reflection $S_{\infty}^{(\mathrm{B})}\left(\eta_{z}\right)=$ $E_{h}\left(\eta_{z} ; z=0\right)$ and amplitude transmission $T_{\infty}^{(\mathrm{B})}\left(\eta_{z}\right)=$ $E_{0}\left(\eta_{z} ; z=L_{z}\right)$ coefficients, one can obtain (Punegov, 1991, 1993; Punegov et al., 2010), in the case of symmetrical Bragg diffraction, the amplitude reflection coefficient at the top surface and the amplitude transmission coefficient at the bottom surface, respectively:

$$
\begin{gathered}
S_{\infty}^{(\mathrm{B})}\left(\eta_{z}\right)=a_{h}^{(\mathrm{B})}\left\{\exp \left[i \xi^{(\mathrm{B})} L_{z}\right]-1\right\} / Q, \\
T_{\infty}^{(\mathrm{B})}\left(\eta_{z}\right)=\exp \left\{i\left[a_{0}^{(\mathrm{B})}+\xi_{1}^{(\mathrm{B})}\right] L_{z}\right\}\left[\xi^{(\mathrm{B})} / Q\right],
\end{gathered}
$$

where $\xi^{(\mathrm{B})}=\left\{\left[2 a_{0}^{(\mathrm{B})}+\eta_{z}\right]^{2}-4 a_{h}^{(\mathrm{B})} a_{-h}^{(\mathrm{B})}\right\}^{1 / 2}, \quad \xi_{1,2}^{(\mathrm{B})}=\left\{-\left[2 a_{0}^{(\mathrm{B})}+\right.\right.$ $\left.\left.\eta_{z}\right] \pm \xi^{(\mathrm{B})}\right\} / 2$ and $Q=\xi_{1}^{(\mathrm{B})} \exp \left[i \xi^{(\mathrm{B})} L_{z}\right]-\xi_{2}^{(\mathrm{B})}$. The primary extinction length (Authier, 2001) $\Lambda_{\text {ext }}=\lambda\left|\sin \theta_{\mathrm{B}}\right| /\left(C \pi\left|\chi_{h}\right|\right)$ is one of the major characteristic parameters of dynamical diffraction in the Bragg geometry. If the crystal thickness is smaller than the primary extinction length, it is usually assumed (Authier, 2001) that kinematical diffraction will occur. This is not always correct for the lateral crystalline structures (Punegov et al., 2014).

Let us now consider the case of symmetrical Laue diffraction on a plane-parallel crystal having thickness $L_{x}$ (see Fig. 1b). Note that we use the same family of reflecting planes $(h k l)$ as in the Bragg case considered above. As the crystal and the incident wave are of infinite extent in the $z$ direction, the amplitudes of both the transmitted and diffracted waves depend only on the $x$ coordinate. Takagi's equations (Takagi, 1969) for the transmitted and diffracted waves are as follows:

$$
\left\{\begin{array}{l}
\frac{\partial}{\partial x} E_{0}\left(\eta_{x} ; x\right)=i a_{0}^{(\mathrm{L})} E_{0}\left(\eta_{x} ; x\right)+i a_{-h}^{(\mathrm{L})} E_{h}\left(\eta_{x} ; x\right) \\
-\frac{\partial}{\partial x} E_{h}\left(\eta_{x} ; x\right)=i\left[a_{0}^{(\mathrm{L})}+\eta_{x}\right] E_{h}\left(\eta_{x} ; x\right)+i a_{h}^{(\mathrm{L})} E_{0}\left(\eta_{x} ; x\right)
\end{array}\right.
$$

where $\quad a_{0}^{(\mathrm{L})}=\pi \chi_{0} /\left(\lambda\left|\cos \theta_{\mathrm{B}}\right|\right) \quad$ and $\quad a_{h,-h}^{(\mathrm{L})}=C \pi \chi_{h,-h} /$ $\left(\lambda\left|\cos \theta_{\mathrm{B}}\right|\right)$. Here, $\eta_{x}=2 k \sin \left(\theta_{\mathrm{B}}\right) \omega$ is the angular parameter describing the $q_{x}$ scan in reciprocal space for the diffracted wave.

The boundary conditions in the case of symmetrical Laue diffraction are $E_{0}\left(\eta_{x} ; x=0\right)=T=1, E_{h}\left(\eta_{x} ; x=0\right)=0$.

In this diffraction geometry, the amplitude reflection $S_{\infty}^{(\mathrm{L})}\left(\eta_{x}\right)=E_{h}\left(\eta_{x} ; x=L_{x}\right)$ and the amplitude transmission $T_{\infty}^{(\mathrm{L})}\left(\eta_{x}\right)=E_{0}\left(\eta_{x} ; x=L_{x}\right)$ coefficients are considered at a distance $L_{x}$ from the entrance surface (see Fig. 1b). The analytical solutions for the amplitude reflection coefficient and the amplitude transmission coefficients, respectively, are (Punegov \& Pavlov, 1992)

$$
\begin{aligned}
& S_{\infty}^{(\mathrm{L})}\left(\eta_{x}\right)=2 i a_{h}^{(\mathrm{L})} \exp \left\{i\left[a_{0}^{(\mathrm{L})}+\eta_{x} / 2\right] L_{x}\right\} \sin \left[\xi^{(\mathrm{L})} L_{x} / 2\right] / \xi^{(\mathrm{L})}, \\
& T_{\infty}^{(\mathrm{L})}\left(\eta_{x}\right)=\exp \left\{i\left[a_{0}^{(\mathrm{L})}+\eta_{x} / 2\right] L_{x}\right\}\left\{\cos \left[\xi^{(\mathrm{L})} L_{x} / 2\right]\right. \\
& \left.-i \eta_{x} \sin \left[\xi^{(\mathrm{L})} L_{x} / 2\right] / \xi^{(\mathrm{L})}\right\}
\end{aligned}
$$

where $\xi^{(\mathrm{L})}=\left[\eta_{x}^{2}+4 a_{h}^{(\mathrm{L})} a_{-h}^{(\mathrm{L})}\right]^{1 / 2}$. In the case of Laue diffraction the major characteristic parameter is the Pendellösung distance $\Lambda_{0}=\lambda\left|\cos \theta_{\mathrm{B}}\right| /\left(C\left|\chi_{h}\right|\right)$ (Authier, 2001).

\section{Acknowledgements}

This study was supported in part by the Ural branch of the Russian Academy of Sciences (project No. 15-9-1-13) and the Russian Foundation for Basic Research (project No. 16-43110350). KMP acknowledges financial support from the University of New England.

\section{References}

Afanas'ev, A. M. \& Kohn, V. G. (1971). Acta Cryst. A27, 421-430.

Authier, A. (2001). Dynamical Theory of X-ray Diffraction. Oxford University Press.

Authier, A., Malgrange, C. \& Tournarie, M. (1968). Acta Cryst. A24, 126-136.

Becker, P. (1977). Acta Cryst. A33, 243-249.

Becker, P. \& Dunstetter, F. (1984). Acta Cryst. A40, 241-251.

Born, M. \& Wolf, E. (1999). Principles of Optics, 7th ed. Cambridge University Press.

Bowen, D. K. \& Tanner, B. K. (1998). High Resolution X-ray Diffractometry and Topography. London: Taylor and Francis.

Cha, W., Song, S., Jeong, N. C., Harder, R., Yoon, K. B., Robinson, I. K. \& Kim, H. (2010). New J. Phys. 12, 035022.

Chukhovskii, F. N., Hupe, A., Rossmanith, E. \& Schmidt, H. (1998). Acta Cryst. A54, 191-198.

Colinge, J.-P., Lee, C.-W., Afzalian, A., Akhavan, N. D., Yan, R., Ferain, I., Razavi, P., O’Neill, B., Blake, A., White, M., Kelleher, A.-M., McCarthy, B. \& Murphy, R. (2010). Nat. Nanotechnol. 5, 225-229.

Dasgupta, N. P., Sun, J., Liu, C., Brittman, S., Andrews, S. C., Lim, J., Gao, H., Yan, R. \& Yang, P. (2014). Adv. Mater. 26, 2137-2184.

Epelboin, Y. (1985). Mater. Sci. Eng. 73, 1-43.

Iida, A. \& Kohra, K. (1979). Phys. Status Solidi A, 51, 533-542.

Jesson, D. E., Pavlov, K. M., Morgan, M. J. \& Usher, B. F. (2007). Phys. Rev. Lett. 99, 016103.

Kaganer, V. M. \& Belov, A. Y. (2012). Phys. Rev. B, 85, 125402.

Kato, N. (1961a). Acta Cryst. 14, 526-532.

Kato, N. (1961b). Acta Cryst. 14, 627-636.

Kato, N. (1963). J. Phys. Soc. Jpn, 18, 1785-1791.

Kato, N. (1964a). J. Phys. Soc. Jpn, 19, 67-77.

Kato, N. (1964b). J. Phys. Soc. Jpn, 19, 971-985. 
Kirste, L., Pavlov, K. M., Mudie, S. T., Punegov, V. I. \& Herres, N. (2005). J. Appl. Cryst. 38, 183-192.

Köhl, M., Schroth, P., Minkevich, A. A. \& Baumbach, T. (2013). Opt. Express, 21, 27734-27749.

Kolosov, S. I. \& Punegov, V. I. (2005). Crystallogr. Rep. 50, 357362.

Krogstrup, P., Jørgensen, H. I., Heiss, M., Demichel, O., Holm, J. V., Aagesen, M., Nygard, J. \& Fontcuberta i Morral, A. (2013). Nat. Photon. 7, 306-310.

Lang, A. R., Kowalski, G. \& Makepeace, A. P. W. (1990). Acta Cryst. A46, 215-227.

Lang, A. R., Kowalski, G., Makepeace, A. P. W. \& Moore, M. (1986). Acta Cryst. A42, 501-510.

Lee, K., Yi, H., Park, W.-H., Kim, Y. K. \& Baik, S. (2006). J. Appl. Phys. 100, 051615

Lehmann, K. \& Borrmann, G. (1967). Z. Kristallogr. 125, 234-248.

Li, Y., Qian, F., Xiang, J. \& Lieber, C. M. (2006). Mater. Today, 9, 18 27.

Mai, Z. \& Zhao, H. (1989). Acta Cryst. A45, 602-609.

Minkevich, A. A., Fohtung, E., Slobodskyy, T., Riotte, M., Grigoriev, D., Metzger, T., Irvine, A. C., Novák, V., Holý, V. \& Baumbach, T. (2011). Europhys. Lett. 94, 66001.

Olekhnovich, N. M. \& Olekhnovich, A. I. (1978). Acta Cryst. A34, 321-326.

Olekhnovich, N. M. \& Olekhnovich, A. I. (1980). Acta Cryst. A36, $22-$ 27.

Pavlov, K. M., Kewish, C. M., David, J. R. \& Morgan, M. J. (2001). J. Phys. D Appl. Phys. 34, A168-A172.

Pavlov, K. M., Punegov, V. I. \& Faleev, N. N. (1995). JETP, 80, 10901097.

Pellegrini, C. \& Stöhr, J. (2003). Nucl. Instrum. Methods Phys. Res. Sect. A, 500, 33-40.

Pietsch, U., Holy, V. \& Baumbach, T. (2004). High-Resolution X-ray Scattering. From Thin Films to Lateral Nanostructures, 2nd ed. New York: Springer-Verlag.

Punegov, V. I. (1991). Sov. Phys. Solid State, 33, 136-140.

Punegov, V. I. (1993). Phys. Status Solidi A, 136, 9-19.

Punegov, V. I. \& Kolosov, S. I. (2007). Crystallogr. Rep. 52, 191-198.
Punegov, V. I., Kolosov, S. I. \& Pavlov, K. M. (2006). Tech. Phys. Lett. 32, 809-812.

Punegov, V. I., Kolosov, S. I. \& Pavlov, K. M. (2014). Acta Cryst. A70, 64-71.

Punegov, V. I., Maksimov, A. I., Kolosov, S. I. \& Pavlov, K. M. (2007). Tech. Phys. Lett. 33, 125-127.

Punegov, V. I., Nesterets, Y. I. \& Roshchupkin, D. V. (2010). J. Appl. Cryst. 43, 520-530.

Punegov, V. I. \& Pavlov, K. M. (1992). Sov. Tech. Phys. Lett. 18, 390391.

Punegov, V. I., Pavlov, K. M., Podorov, S. G. \& Faleev, N. N. (1996). Phys. Solid State, 38, 148-152.

Saka, T., Katagawa, T. \& Kato, N. (1972a). Acta Cryst. A28, 102-113.

Saka, T., Katagawa, T. \& Kato, N. (1972b). Acta Cryst. A28, 113-120.

Saka, T., Katagawa, T. \& Kato, N. (1973). Acta Cryst. A29, 192-200.

Saldin, D. K. (1982). Acta Cryst. A38, 425-432.

Stankevič, T., Dzhigaev, D., Bi, Z., Rose, M., Shabalin, A., Reinhardt, J., Mikkelsen, A., Samuelson, L., Falkenberg, G., Vartanyants, I. A. \& Feidenhans'l, R. (2015). Appl. Phys. Lett. 107, 103101.

Stepanov, S. \& Forrest, R. (2008). J. Appl. Cryst. 41, 958-962.

Takagi, S. (1962). Acta Cryst. 15, 1311-1312.

Takagi, S. (1969). J. Phys. Soc. Jpn, 26, 1239-1253.

Taupin, D. (1967). Acta Cryst. 23, 25-35.

Thorkildsen, G. \& Larsen, H. B. (1999a). Acta Cryst. A55, 840-854.

Thorkildsen, G. \& Larsen, H. B. (1999b). Acta Cryst. A55, 1-13.

Uragami, T. S. (1969). J. Phys. Soc. Jpn, 27, 147-154.

Uragami, T. S. (1970). J. Phys. Soc. Jpn, 28, 1508-1527.

Uragami, T. S. (1971). J. Phys. Soc. Jpn, 31, 1141-1161.

Uragami, T. S. (1983). J. Phys. Soc. Jpn, 52, 3073-3079.

Vartanyants, I. A. \& Yefanov, O. M. (2015). X-ray Diffraction: Modern Experimental Techniques, edited by O. H. Seek \& B. M. Murphy, ch. 12. Boca Raton: Pan Stanford Publishing.

Yan, R., Gargas, D. \& Yang, P. (2009). Nat. Photon. 3, 569-576.

Yan, H. \& Li, L. (2014). Phys. Rev. B, 89, 014104.

Yan, H. \& Noyan, I. C. (2005). J. Appl. Phys. 98, 073527.

Yan, H. \& Noyan, I. C. (2006). J. Appl. Cryst. 39, 320-325.

Yang, W., Huang, X., Harder, R., Clark, J. N., Robinson, I. K. \& Mao, H. K. (2013). Nat. Commun. 4, 1680. 